\title{
CLASSIFICATION OF COMPACT HOMOGENEOUS SPACES WITH INVARIANT $G_{2}$-STRUCTURES
}

\author{
HÔNG VÂN LÊ AND MOBEEN MUNIR
}

\begin{abstract}
In this note we classify all homogeneous spaces $G / H$ admitting a $G$-invariant $G_{2}$-structure, assuming that $G$ is a connected compact Lie group and $G$ acts effectively on $G / H$. They include a subclass of all homogeneous spaces $G / H$ with a $G$-invariant $\tilde{G}_{2}$-structure, where $G$ is a compact Lie group. There are many new examples with nontrivial fundamental group. We study a subclass of homogeneous spaces of high rigidity and low rigidity and show that they admit families of invariant coclosed $G_{2}$-structures (resp. $\tilde{G}_{2}$-structures).
\end{abstract}

\section{Contents}

1. Introduction

2. The groups $\tilde{G}_{2}$ and $G_{2}$

2.1. The group $\tilde{G}_{2}$ and its maximal compact subgroup $S O(4)$

2.2. The group $G_{2}$ and its maximal compact subgroups

3. Compact homogeneous manifolds admitting invariant $\tilde{G}_{2}$-structures

3.1. Reduction to a representation problem

3.2. Classification theorem

4. Compact homogeneous manifolds admitting invariant $G_{2}$-structures

4.1. Reduction to a representation problem

4.2. Classification theorem

5. Spaces $G / H$ with high rigidity or with low rigidity 20

5.1. Spaces $G / H$ with $d_{3}(G / H)=1$

5.2. Spaces $G / H$ with $d_{3}(G / H)=2$

5.3. Spaces with $d_{3}(G / H)=35$

References

MSC: $57 M 50,57 M 60$

Keywords: compact homogeneous space, $G_{2}$-structure

\section{InTRODUCTION}

In recent years manifolds admitting a $G_{2}$-structure have attracted increasing interests of physicists and mathematicians. These manifolds can be geometric models in the theory of superstrings with torsion [12. In another field, a recent work of

H.V.L. is partially supported by grant of ASCR Nr IAA100190701 and M.M. is partially supported by HEC of Pakistan. 
Donaldson and Segal [9] suggests that a right framework for a gauge theory in dimension 7 is a class of manifolds with non-vanishing torsion $G_{2}$-structure. A main source of computable models of manifolds with $G_{2}$-structures are homogeneous spaces or spaces of co-homogeneity one [17, [6], 8].

In this note we classify all compact homogeneous spaces $M^{7}$ of the form $G / H$ such that $G$ is a connected compact Lie group acting effectively on $G / H$, admitting a $G$-invariant structure of $G_{2}$-type or of the non-compact form $\tilde{G}_{2}$-type. This classification extends the classification by Friedrich-Kath-Moroianu-Semmelmann of all simply-connected compact homogeneous nearly parallel $G_{2}$-manifolds in [11. We study manifolds with $\tilde{G}_{2}$-structure, not only because of their striking similarity with those admitting a $G_{2}$-structure, but they present an interesting class in pseudo Riemannian geometry [19. We also like to point out that even the classification of symmmetric spaces with holonomy contained in $\tilde{G}_{2}$ is open.

Recall that a 7-dimensional smooth manifold $M^{7}$ is said to admit $a G_{2}$-structure (resp. a $\tilde{G}_{2}$-structure), if there is a section of the bundle $\mathcal{F}\left(M^{7}\right) / G_{2}\left(\right.$ resp. $\left.\mathcal{F}\left(M^{7}\right) / \tilde{G}_{2}\right)$ over $M^{7}$, where $\mathcal{F}\left(M^{7}\right)$ is the frame bundle over $M^{7}$. It is well-known that $G_{2}$ (resp. $\left.\tilde{G}_{2}\right)$ is the automorphism group of a 3 -form $\phi(\operatorname{resp} . \tilde{\phi})$ on $\mathbb{R}^{7},[25$, [13, p. 114], or [3, p. 539]. Such a 3-form $\phi$ (resp. $\tilde{\phi}$ ) is called a 3-form of $G_{2}$-type (resp. $\tilde{G}_{2}$-type). It is known that the $G L\left(\mathbb{R}^{7}\right)$-orbits of $\phi$ and $\tilde{\phi}$ are the only open orbits of the $G L\left(\mathbb{R}^{7}\right)$-action on $\Lambda^{3}\left(\mathbb{R}^{7}\right)^{*}$, see e.g. 3], [15, 22. Any 3 -form on these open orbits is called a stable 3-form, [15, or a definite 3-form, if it lies in the orbit of $\phi$, or an indefinite 3-form, if it lies in the orbit of $\tilde{\phi}$. The existence of a $G_{2}$-structure (resp. $\tilde{G}_{2}$-structure) on a manifold $M^{7}$ is equivalent to the existence of a definite differential 3-form $\phi$ (resp. indefinite differential 3-form $\tilde{\phi}$ ) on $M^{7}$.

The plan of our note is as follows. In section 2 we collect important properties of the groups $\tilde{G}_{2}$ and $G_{2}$, which are needed for our classification. In section 3 we classify homogeneous manifolds $G / H$ admitting invariant $\tilde{G}_{2}$-structures, where $G$ is a connected compact Lie group and $H$ is a closed Lie subgroup (not necessary connected) of $G$, see Theorem 3.2.1. This problem is equivalent to finding all pairs $(G, H)$ where $H$ is a closed (hence compact) subgroup of a compact Lie group $G$ such that the image of the isotropy representation $\rho(H)$ is a subgroup of $\tilde{G}_{2} \subset G l(7, \mathbb{R})$. We observe that any such homogeneous space $G / H$ also admits an invariant $G_{2^{-}}$ structure, hence $\rho(H)$ is also a subgroup of $G_{2} \subset G l(7, \mathbb{R})$. In section 4 we classify all homogeneous manifolds $G / H$ admitting invariant $G_{2}$-structures, where $G$ is a compact Lie group and $H$ is a closed Lie subgroup (not necessary connected) of $G$, see Theorem 4.2.1. Our classification is reduced to finding all pairs $(G, H)$ such that the image of the isotropy representation $\rho(H)$ is a subgroup of $G_{2} \subset G l(7, \mathbb{R})$. We also compute the dimension of the space of all $G$-invariant $G_{2}$-structures on a homogeneous manifold $G / H$, see Remark 4.2.2 a. In section 5 we study a special class of homogeneous manifolds $G / H$ admitting invariant $G_{2}$-structures using our classification. Among these spaces there are many known examples of manifolds admitting $G_{2}$-structures. We explain some known properties of these examples using simpler arguments based on our classification. We also present some new results concerning these spaces.

Let us describe the method of our classification. First we notice that $G / H$ admits a $G$-invariant $G_{2}$-structure (resp. $\tilde{G}_{2}$-structure), if and only if it admits a $G$ invariant definite 3 -form (resp. indefinite 3 -form). In the first step we find all pairs of corresponding Lie algebras $(\mathfrak{h} \subset \mathfrak{g})$. In the second step we find the associated 
pairs of Lie groups $(H \subset G)$. The first step is done using representation theory and is fairly standard, even it could be done using some special software package. There is no algorithm known to solve the second problem. So we have developed a set of techniques to find the normalizer of a given connected Lie subgroup, and after that we can find all Lie subgroups (not necessary connected) with a given Lie algebra obtained in the first step.

Finally we remark that the problem we solve in this note is a part of a more general question to classify all homogeneous spaces $M=G / H$ admitting $G$-invariant $\tilde{G}_{2}$-structures or $G$-invariant $G_{2}$-structures. If we require $M$ to be compact and with finite fundamental group, by the Montgomery theorem [23, Corollary 3], $M$ has also a transitive action of a compact subgroup $G^{\prime} \subset G$. Thus $G$ is a subgroup of the full diffeomorphism group of $M=G^{\prime} /\left(G^{\prime} \cap H\right)$ preserving a given $G^{\prime}$-invariant $\tilde{G}_{2^{-}}\left(\right.$resp. $\left.G_{2^{-}}\right)$structure on $M$.

\section{The GROUPS $\tilde{G}_{2}$ AND $G_{2}$}

In this section we recall the definitions of $\tilde{G}_{2}$ and $G_{2}$. We describe the maximal compact subgroup of $\tilde{G}_{2}$, which is unique up to conjugacy by elements of $\tilde{G}_{2}$. We also describe maximal compact subgroups of $G_{2}$. These subgroups are needed for our classifications in sections 3 and 4.

2.1. The group $\tilde{G}_{2}$ and its maximal compact subgroup $S O(4)$. We refer the reader to [3] for a definition and properties of the exceptional Lie group $\tilde{G}_{2}$. For the convenience of the reader we briefly describe the group $\tilde{G}_{2}$, which is less familiar than its dual compact group $G_{2}$.

Let us fix a basis $e^{1}, \cdots, e^{7}$ in $\left(\mathbb{R}^{7}\right)^{*}$. Denote by $\omega^{i j k}$ the 3 -form $e^{i} \wedge e^{j} \wedge e^{k} \in$ $\Lambda^{3}\left(\mathbb{R}^{7}\right)^{*}$.

Definition 2.1.1. 25], see also 3, Definition 2, p.543]. The group $\tilde{G}_{2}$ is defined as the subgroup $\left\{g \in G L\left(\mathbb{R}^{7}\right) \mid g^{*}(\tilde{\phi})=\tilde{\phi}\right\}$ where

$$
\tilde{\phi}=\omega^{123}-\omega^{145}-\omega^{167}-\omega^{246}+\omega^{257}+\omega^{347}+\omega^{356} .
$$

Lemma 2.1.2. [3, Theorem 2] The group $\tilde{G}_{2}$ is the automorphism group of the split-octonion algebra. The group $\tilde{G}_{2}$ is connected.

Theorem 2 in 3 . cited above is given without a proof (but it can be proved in the same way as in the proof of 3. Theorem 1]). A similar explanation for the first assertion of Lemma 2.1.2 can be found in [22, §6.2], where we proved that $\tilde{G}_{2}$ is a subgroup of the automorphism group of the Malcev simple algebra of dimension 7 , which is the imaginary part $\operatorname{Im} \mathbb{O}_{S}$ of the split-octonion algebra $\mathbb{O}_{S}$. Since the multiplication on the Malcev algebra is the imaginary part of the octonion multiplication on $\operatorname{Im} \mathbb{O}_{S}$, we get easily $\tilde{G}_{2} \subset \operatorname{Aut}\left(\mathbb{O}_{S}\right)$. The other inclusion $\operatorname{Aut}\left(\mathbb{O}_{S}\right) \subset \tilde{G}_{2}$ can be verified straightforwardly. A detailed proof for the second assertion of Lemma 2.1.2 can be found in 21. (the first version, which is also available at the arxiv server), namely this assertion is a direct consequence of Lemmas 2.1 and 2.2 proved therein.

As a topological space, $\tilde{G}_{2}$ is a direct product of its maximal compact Lie subgroup and a vector space. 
Lemma 2.1.3. The maximal compact subgroup of $\tilde{G}_{2}$ is $S O(4)$. The inclusion of $S O(4) \rightarrow \tilde{G}_{2} \rightarrow G l\left(\mathbb{R}^{7}\right)$ acts on $\mathbb{R}^{7}$ with two irreducible subspaces of dimension 3 and dimension 4. Any compact subgroup of $\tilde{G}_{2}$ is conjugate to a subgroup in the maximal compact subgroup $S O(4)$.

The first assertion of Lemma 2.1 .3 is known to experts in the Cartan theory of real semisimple Lie groups but we don't find an explicit proof of it in standard text-books. In [21, Corollary 2.4] we give a topological proof of this assertion. For the convenience of the reader we give here another algebraic proof, which explains also the second assertion of Lemma 2.1.3. By [14, Theorem 1.1, p.252] the maximal compact Lie subgroup of $\tilde{G}_{2}$ is connected whose Lie algebra is a maximal compact Lie subalgebra in $\tilde{\mathfrak{g}}_{2}$. Note that $\mathfrak{s} o(4)=\mathfrak{s} u(2)+\mathfrak{s} u(2)$ is a maximal compact Lie subalgebra of $\tilde{\mathfrak{g}}_{2}$ which can be described in terms of the root decomposition of the complex Lie algebra $\mathfrak{g}_{2}^{\mathbb{C}}$, namely it is the intersection of the normal form $\tilde{\mathfrak{g}}_{2}$ of $\mathfrak{g}_{2}^{\mathbb{C}}$ and the compatible compact form $\mathfrak{g}_{2}$. Using the weights of the representation of the subalgebra $\mathfrak{s} u(2)+\mathfrak{s} u(2) \subset \tilde{\mathfrak{g}}_{2}$ on $\mathbb{R}^{7}$, it is easy to see that the corresponding connected Lie subgroup in $\tilde{G}_{2}$ is $S O(4)$ and the corresponding representation is a sum of two real irreducible representations of dimension 3 and dimension 4 . This proves the first and the second assertion of Lemma 2.1.3. The last assertion of Lemma 2.1.3 is a consequence of [14, Theorem 2.1, p. 256].

We now describe another way to construct an explicit embedding of $S O(4)$ into $\tilde{G}_{2}$, see [13, chapter IV,(1.9), p. 115], since it will be useful in our computations later. The group $S p(1) \times S p(1)$ acts on the split-octonion algebra $\mathbb{O}_{S}=\mathbb{H} \oplus \mathbb{H} e$ as follows:

$$
\chi\left(q_{1}, q_{2}\right)(a+b e):=\left(q_{1} a \bar{q}_{1}+q_{2} b \bar{q}_{1} e\right) .
$$

It is easy to see that this action defines an embedding of $S O(4)$ into $\tilde{G}_{2}$. Thus we can regard this maximal compact subgroup $S O(4)$ as the intersection $\tilde{G}_{2} \cap(S O(\operatorname{Im} \mathbb{H}) \times$ $S O(\mathbb{H} e)$ ). Taking into account [14, Theorem 1.1, p.252] this construction also gives a proof of the first and the second assertion of Lemma 2.1.3.

To distinguish an abstract Lie group $S O(4)$ (resp. a Lie algebra $\mathfrak{s o ( 4 )}$ ) with its image inside $\tilde{G}_{2}$ (resp. $\tilde{\mathfrak{g}}_{2}$ ) we denote the later one by $S O(4)_{3,4}\left(\right.$ resp. $\left.\mathfrak{s o}(4)_{3,4}\right)$. Note that the conjugacy class of $S O(4)_{3,4}$ in $G l\left(\mathbb{R}^{7}\right)$ is defined uniquely by the highest weights of its representation. We denote by $\mathfrak{s} u(2)_{3,4}$ the Lie subalgebra in $\mathfrak{s o}(4)_{3,4}$ corresponding to the Lie subgroup $\left\{\chi\left(q_{1}, 1\right) \mid q_{1} \in S p(1)\right\}$ in formula (2.1), and by $\mathfrak{s} u(2)_{0,4}$ the Lie subalgebra corresponding to the Lie subgroup $\left\{\chi\left(1, q_{2}\right) \mid, q_{2} \in S p(1)\right\}$. The conjugacy of $\mathfrak{s o}(4)_{3,4}=\mathfrak{s} u(2)_{3,4}+\mathfrak{s} u(2)_{2,4}$ in $g l\left(\mathbb{R}^{7}\right)$ is defined uniquely up to conjugacy by the highest weights $(2,0)$ and $(1,1)$ of the irreducible components of the representation of $\mathfrak{s o}(4)$ explained in Lemma 2.1.3 and in (2.1). The weight $(2,0)$ corresponds to the irreducible real representation of dimension 3 , and the weight $(1,1)$ corresponds to the irreducible real representation of dimension 4. We refer the reader to [24, §8] for a comprehensive exposition of the theory of real representations of real semisimple Lie algebras, or 28, Appendix] for a compact exposition of the theory. Since $S O(4)$ is connected, the conjugacy class of an embedding $S O(4) \rightarrow G l\left(\mathbb{R}^{7}\right)$ is defined uniquely by the representation of its Lie algebra $\mathfrak{s} u(2)_{1}+\mathfrak{s} u(2)_{2}$, where $\mathfrak{s} u(2)_{1}\left(\right.$ resp. $\left.\mathfrak{s} u(2)_{2}\right)$ is the Lie algebra of the first (resp. the second) subgroup $S p(1)$ defined just before (2.1).

We also remark that there are three non-conjugate subalgebras in $\mathfrak{s o}(4)_{3,4}$ which are isomorphic to $\mathfrak{s} o(3)=\mathfrak{s} u(2)$. We denote by $\mathfrak{s} o(3)_{3,3}$ the third Lie subalgebra 
in this subclass. It is defined by the diagonal embedding of $\mathfrak{s o}(3)=\mathfrak{s} u(2)$ into $\mathfrak{s o}(4)_{3,4}=\mathfrak{s} u(2)_{3,4}+\mathfrak{s} u(2)_{0,4}$.

We summarize a part of our discussion in the following

Lemma 2.1.4. The image of a representation $\bar{\chi}: S O(4) \rightarrow G l\left(\mathbb{R}^{7}\right)$ is conjugate to $S O(4)_{3,4} \subset G l\left(\mathbb{R}^{7}\right)$, if and only $\bar{\chi}$ is a sum of two irreducible real representations, one of dimension 3 with the highest weight $(2,0)$, and the other of dimension 4 with the highest weight $(1,1)$.

\subsection{The group $G_{2}$ and its maximal compact subgroups.}

Definition 2.2.1. [25], see also [13, IV.1.A, p.114], and [3, Definition 1, p.539]. The group $G_{2}$ is defined as the subgroup $\left\{g \in G L\left(\mathbb{R}^{7}\right) \mid g^{*}(\tilde{\phi})=\tilde{\phi}\right\}$ where

$$
\phi=\omega^{123}+\omega^{145}+\omega^{167}+\omega^{246}-\omega^{257}-\omega^{347}-\omega^{356} .
$$

We observe that $\phi+\tilde{\phi}=2 \omega^{123}$.

Dynkin's classical result 10 asserts that the Lie algebra $\mathfrak{g}_{2}$ has exactly three (up to conjugation) maximal subalgebras of dimensions 8,6 and 3 respectively: $\mathfrak{s} u(3), \mathfrak{s o}(4)_{3,4}, \mathfrak{s o}(3)_{7}$, from which we have seen $\mathfrak{s o}(4)_{3,4}$ in the previous subsection. The Lie subalgebra $\mathfrak{s u}(3)$ is the intersection $\mathfrak{g}_{2} \cap \mathfrak{g} l\left(\mathbb{R}^{6}\right) \subset \mathfrak{g l}\left(\mathbb{R}^{7}\right)$ for any embedding $\mathfrak{g l}\left(\mathbb{R}^{6}\right) \subset \mathfrak{g} l\left(\mathbb{R}^{7}\right)$, see e.g. [8, $\left.\S 2\right]$ for a proof. The Lie subalgebra $\mathfrak{s o}(3)_{7}$ is defined by a real irreducible representation of $\mathfrak{s} u(2)$ of real dimension 7 .

Let us fix the basis $\left(e_{i}\right)$ of $\mathbb{R}^{7}$ dual to the basis $\left(e^{i}\right)$. Denote by $D_{7}$ the element $\operatorname{diag}(-1,1,-1,1,-1,1,-1) \in G l\left(\mathbb{R}^{7}\right)$. It is easy to check that $D_{7}$ preserves the form $\phi$, hence $D_{7} \in G_{2}$.

For any element $a$ of order $k$ in a group $G$ we denote by $\mathbb{Z}_{k}[a]$ the cyclic subgroup in $G$ generated by $a$.

Lemma 2.2.2. Any maximal proper subgroup in $G_{2}$ is conjugate to one of the following subgroups in $G_{2}: S U(3) \cdot \mathbb{Z}_{2}\left[D_{7}\right], S O(4)_{3,4}, S O(3)_{7}$.

This Lemma is likely known to experts (see e.g. [8, §8, p.112] for a statement without a proof that the normalizer $\mathcal{N}_{G_{2}}(S U(3))$ is $\left.S U(3) \cdot \mathbb{Z}_{2}\left[D_{7}\right]\right)$, but we do not have a reference with a proof of it. For the convenience of the reader we give here a proof of Lemma 2.2.2 using the Dynkin result above, combining with the invariance principle as well as with the Schur's Lemma and its consequence stated below.

- Invariance principle. Suppose that $H^{0}$ is a (connected) subgroup of $G \subset$ $S O(W)$. We denote by $U$ the fixed-point subspace of the action of $H^{0}$ on $W$. Then the normalizer $\mathcal{N}_{G}\left(H^{0}\right)$ preserves the subspace $U$ and its orthogonal complement $U^{\perp}$.

- Schur's Lemma and its consequence. Suppose that the inclusion $H^{0} \rightarrow G \rightarrow$ $G l\left(\mathbb{R}^{n}\right) \rightarrow G l\left(\mathbb{C}^{n}\right)$ gives a complex irreducible representation of $H^{0}$ in $G l\left(\mathbb{C}^{n}\right)$. Then the centralizer $\mathcal{Z}_{G}\left(H^{0}\right)$ is equal to the center $\mathcal{Z}(G)$ of $G$. Using this we can compute $\mathcal{N}_{G}\left(H^{0}\right)$ easily, taking into account the relation $\operatorname{Int}\left(H^{0}\right) \subset \mathcal{N}_{G}\left(H^{0}\right) / \mathcal{Z}_{G}\left(H^{0}\right) \subset$ Aut $\left(H^{0}\right)$.

Applying the invariance principle to $W=\mathbb{R}^{7}, U=\mathbb{R}$, we conclude that if $x \in$ $\mathcal{N}_{G_{2}}(S U(3))$ then either $x \in G l\left(\mathbb{R}^{6}\right) \cap G_{2}=S U(3)$ or $x \cdot D_{7} \in G l\left(\mathbb{R}^{6}\right) \cap G_{2}=S U(3)$, what proves the first assertion. To compute $\mathcal{N}_{G_{2}}\left(S O(4)_{3,4}\right)$ we apply the invariance principle to the space $W=\left(\Lambda^{3}\left(\mathbb{R}^{7}\right)^{*}\right)_{\phi}^{\perp}$ which is the orthogonal complement to $\langle\phi\rangle_{\mathbb{R}}$ in $\Lambda^{3}\left(\mathbb{R}^{7}\right)^{*}$. Note that $\phi_{0}=\phi-7 \omega^{123}$ is an element of $W$, and $U=\left\langle\phi_{0}\right\rangle_{\mathbb{R}}$ is the fixed point subspace of the induced $S O(4)_{3,4}$-action on $W$. By the invariance 
principle $U$ is invariant under the induced action of $\mathcal{N}_{G_{2}}\left(S O(4)_{3,4}\right)$. Note that for $g \in \mathcal{N}_{G_{2}}\left(S O(4)_{3,4}\right)$, we have $g^{*}\left(\phi_{0}\right)= \pm \phi_{0}$, since $g \in S O(7)$. If $g^{*}\left(\phi_{0}\right)=\phi_{0}$, then $g$ must belong to $S O(4)_{3,4}$. If not, then $g^{*}\left(7 \omega^{123}\right)=2 \phi-7 \omega^{123}$. Taking into account that $g$ preserves the induced norm on $W \subset \Lambda^{3}\left(\mathbb{R}^{7}\right)^{*}$, we obtain a contradiction. Hence $\mathcal{N}_{G_{2}}\left(S O(4)_{3,4}\right)=S O(4)_{3,4}$. Using the Schur's Lemma and its consequence, taking into account that $\mathcal{Z}\left(G_{2}\right)=\mathbb{Z}_{1}[14$, p.516], we conclude that the normalizer $\mathcal{N}_{G_{2}}\left(S O(3)_{7}\right)$ is $S O(3)_{7}$, the connected Lie subgroup having Lie algebra $\mathfrak{s o}(3)_{7}$.

\section{COMPACT HOMOGENEOUS MANIFOLDS ADMitTing INVARIANT $\tilde{G}_{2}$-STRUCTURES}

In this section we classify homogeneous manifolds $G / H$ admitting $G$-invariant $\tilde{G}_{2}$-structures, where $G$ is a compact Lie group and $H$ is a closed Lie subgroup (not necessary connected) of $G$. Since $H$ is a compact Lie group, this problem is equivalent to the classification of all pairs $(G, H)$ such that the image of the isotropy representation $\rho(H)$ is a compact subgroup of $\tilde{G}_{2} \subset G l(7, \mathbb{R})$. In subsection 3.1 we reduce the classification problem to a representation problem, which is essentially linear when we classify only the corresponding Lie algebras $(\mathfrak{g}, \mathfrak{h})$. The hardest part is to find all disconnected closed Lie subgroups $H$ whose isotropy representation maps $H$ into a subgroup of $\tilde{G}_{2}$. In subsection 3.2 we summarize our classification in a table. We also compute the dimension of the space of $G$-invariant $\tilde{G}_{2}$-structures on each manifold $G / H$.

3.1. Reduction to a representation problem. In this subsection we first find Lie algebras $(\mathfrak{h} \subset \mathfrak{g})$ of compact Lie groups $(H \subset G)$ such that $(G / H)$ admits a $G$ invariant $\tilde{G}_{2}$-structure, and then we find the corresponding pairs $(H \subset G)$. Though the first step is a standard technique, we describe all these algebras in detail, since we use this description in the second step.

Let $G$ be a connected compact Lie group which acts transitively on a connected compact smooth manifold $M^{7}=G / H$. Without lost of generality we can assume that $G$ acts effectively on $M$.

Let $\langle,\rangle_{\mathfrak{g}}$ be a left and right invariant metric on $G$. Denote by $\rho$ the isotropy representation of $H$ on the tangent space $T_{e H} G / H=\mathbb{R}^{7}$. Let $\mathfrak{g}$ (resp. $\mathfrak{h}$ ) be the Lie algebra of $G$ (resp. $H$ ). We write $\mathfrak{g}=\mathfrak{h}+V$, where $V$ is the orthogonal complement to $\mathfrak{h}$ w.r.t. $\langle,\rangle_{\mathfrak{g}}$. Denote by $\bar{\rho}$ the induced isotropy action of $\mathfrak{h}$ on $V$. Since the action of $G$ is almost effective, $\operatorname{ker} \bar{\rho}=0$.

Taking into account Lemma 2.1.3 and our discussion at the end of subsection 2.1 we get immediately

Lemma 3.1.1. $G / H$ admits a $G$-invariant $\tilde{G}_{2}$-structure if and only if $\rho(H)$ lies in a compact subgroup $S O(4)_{3,4} \subset G l(V)$. Consequently, the Lie subalgebra $\bar{\rho}(\mathfrak{h}) \subset$ $\mathfrak{s o}(4)_{3,4}$ is one of the following subalgebras

1) $\bar{\rho}(\mathfrak{h})=\mathfrak{s o}(4)_{3,4}$; (we shall use "=", "be", "coincide with", "equal to" for "be conjugate to", if no misunderstanding arises).

2) $\bar{\rho}(\mathfrak{h})=\mathfrak{s o}(3)$ with three possible embeddings into $\mathfrak{s o}(4)_{3,4}$ :

(2a) $-\bar{\rho}(\mathfrak{h})=s o(3)_{3,3}$;

$(2 b)-\bar{\rho}(\mathfrak{h})=\mathfrak{s} u(2)_{3,4}$

(2c) $-\bar{\rho}(\mathfrak{h})=\mathfrak{s} u(2)_{0,4}$. 
3) $\bar{\rho}(\mathfrak{h})=\mathfrak{s o}(3)+\mathbb{R}$ with two possible embeddings into $\mathfrak{s o}(4)_{3,4}$;

(3a) - the summand $\mathfrak{s o}(3) \subset \bar{\rho}(\mathfrak{h})$ coincides with $\mathfrak{s} u(2)_{3,4}$,

(3b) - the summand $\mathfrak{s o}(3) \subset \bar{\rho}(\mathfrak{h})$ coincides with $\mathfrak{s} u(2)_{0,4}$.

4) $\bar{\rho}(\mathfrak{h})=\mathbb{R}^{2}$.

5) $\bar{\rho}(\mathfrak{h})=\mathbb{R}^{1}=\mathfrak{s o}(2)$ (there are infinitely many inequivalent embeddings of $\mathfrak{s o}(2)$ into $\mathfrak{s o}(4))$.

6) $\bar{\rho}(\mathfrak{h})=0$.

Let us explain our method to find all pairs $(H \subset G)$ satisfying the conditions in our classification.

By Levy decomposition theorem we can represent $G$ as a quotient $\left(G^{s c} \times T^{k}\right) / Z$, where $G^{s c}$ is a connected simply-connected semisimple compact Lie group and $Z$ is a finite central subgroup of $\hat{G}=G^{s c} \times T^{k}$. Denote by $p$ the projection $\hat{G} \rightarrow G$. Note that the action of $\hat{G}$ on $\hat{G} / p^{-1}(H)$ is almost effective. Moreover the image of the isotropy action of $p^{-1}(H)$ on $V$ coincides with the image of the isotropy action of $H$ on $V$. Hence $\hat{G} / p^{-1}(H)$ admits a $\hat{G}$-invariant $\tilde{G}_{2}$-structure, if $G / H$ does. Next we observe that the effectiveness of the action of $G$ on $G / H$ is equivalent to the relation $\mathcal{Z}(G) \cap H=I d$, assuming that the action of $G$ on $G / H$ is almost effective, i.e. $\operatorname{ker} \bar{\rho}=0$. This is equivalent to the relation $\mathcal{Z}(\hat{G}) \cap p^{-1}(H)=Z$. Under the assumption that $\hat{G}$ acts on $\hat{G} / H^{\prime}$ almost effectively, we reduce a classification of all pairs $H \subset G$ satisfying our conditions to a classification of all pairs $\left(H^{\prime} \subset \hat{G}\right)$ such that $\hat{G} / H^{\prime}$ admits a $\hat{G}$-invariant $\tilde{G}_{2}$-structure. To get the corresponding groups $H \subset G$ we set $G=\hat{G} /\left(\mathcal{Z}(\hat{G}) \cap H^{\prime}\right), H=H^{\prime} /\left(\mathcal{Z}(\hat{G}) \cap H^{\prime}\right)$. We solve this problem in the following steps. In the first step, for each possibility among (1) -(6) above, we find all pairs $(\mathfrak{h} \subset \mathfrak{g})$ of a compact Lie algebra $\mathfrak{h}$ of co-dimension 7 in a compact Lie algebra $\mathfrak{g}$ such that the adjoint representation $\bar{\rho}(\mathfrak{h})$ on $V$ is the given possibility, moreover ker $\bar{\rho}=0$. Then we find a connected Lie subgroup $H^{0} \subset \hat{G}$ with the given Lie algebra $\mathfrak{h} \subset \mathfrak{g}$. As we have mentioned above, this step is fairly standard.

In the second step we find all Lie subgroups $H$ in $\hat{G}$ with Lie algebra $\mathfrak{h}$ obtained in the first step. This subgroup lies in the normalizer $\mathcal{N}_{\hat{G}}\left(H^{0}\right)$. It is an extension of a finite subgroup $\Gamma$ in $\mathcal{N}_{\hat{G}}\left(H^{0}\right) / H^{0}$ by $H^{0}$. In our note we compute the normalizer of a connected Lie subgroup $H^{0}$ in a compact Lie group $G$ by using ad hoc methods for each separate case. The invariance principle as well as the Schur's Lemma and its consequence are also used frequently in our consideration.

In the third step we verify if the isotropy action of this subgroup $H$ on $V$ lifts to an embedding into the group $S O(4)_{3,4} \subset \tilde{G}_{2}$.

In the final step we compute $\mathcal{Z}(\hat{G}) \cap H$, knowing $\mathcal{Z}(\hat{G})=\mathcal{Z}\left(G^{s c}\right) \times T^{k}$. The center $\mathcal{Z}\left(G^{s c}\right)$ is known, see e.g. table 10 in [28].

Now we proceed to consider each possibility listed in Lemma 3.1.1.

Possibility 1 with $\bar{\rho}(\mathfrak{h})=\mathfrak{s o}(4)_{3,4}$. Taking into account Lemma 2.1.4 we conclude that $\mathfrak{g}$ must be semisimple. Since $\operatorname{dim} \mathfrak{g}=13$ and $\mathfrak{g} \supset \mathfrak{s o}(4)$, we conclude that $\mathfrak{g}=\mathfrak{s o} o(5)+\mathfrak{s o} o(3)$.

Proposition 3.1.2. Suppose that $\hat{G} / H$ admits a $\hat{G}$-invariant $\tilde{G}_{2}$-structure such that their corresponding Lie algebras $(\mathfrak{h} \subset \mathfrak{g})$ are in possibility 1 . Then $\hat{G}=G^{s c}=$ $S p(2) \times S p(1)$. The corresponding Lie subgroup $H$ is either $S p(1)_{1} \times S p(1)_{2}$, or the normalizer $S p(1)_{1} \times S p(1)_{2} \times \mathbb{Z}_{2}[\mathcal{Z}(S p(1))]$, described in the proof below. The kernel of the $G^{s c}$-action is $\mathbb{Z}_{2}$, or $\mathbb{Z}_{2} \times \mathbb{Z}_{2}[\mathcal{Z}(S p(1))]$ respectively. 
Proof. In this case the embedding $\Pi: \mathfrak{h}=\mathfrak{s o}(4)=\mathfrak{s o}(3)_{1}+\mathfrak{s o}(3)_{2} \rightarrow \mathfrak{g}=\mathfrak{s o}(5)+$ $\mathfrak{s o}(3)$ is defined as follows. $\Pi$ is a direct sum of the canonical embedding $\Pi^{0}: \mathfrak{h}=$ $\mathfrak{s} p(1)_{1}+\mathfrak{s} p(1)_{2} \rightarrow \mathfrak{s} p(2)=\mathfrak{s o}(5) \subset \mathfrak{g}$ and the projection $\Pi^{1}$ from $\mathfrak{h}$ to the ideal $\mathfrak{s o}(3) \subset \mathfrak{g}$. In this note we use frequently isomorphism $\mathfrak{s} p(1)=\mathfrak{s} o(3)=\mathfrak{s} u(2)$, so $\mathfrak{s} p(1)_{i}$ denotes the same subalgebra $\mathfrak{s o}(3)_{i}, \mathrm{i}=1,2$. The space $V$ is $W+W^{\perp}$, where $W$ is the orthogonal complement of $\Pi^{0}\left(\mathfrak{s} p(1)_{1}+\mathfrak{s} p(1)_{2}\right)$ in $\mathfrak{s} p(2)$ and $W^{\perp}$ is the orthogonal complement of $W$ in $V$. We also denote by $\Pi$ the lift of the representation $\Pi$ to the corresponding simply connected Lie group $G^{s c}$. Let $S p(1)_{i}$ be the corresponding Lie subgroup in $G^{s c}=S p(2) \times S p(1)$ with Lie subalgebra $\mathfrak{s} p(1)_{i}$. Below we decompose $\mathfrak{s} p(2)=\Pi^{0}(\mathfrak{h})+W$ in a matrix expression, cf. [14, p. 446].

$$
\mathfrak{s} p(2)=\left(\begin{array}{cccc}
i a_{1} & w_{1} & z_{1} & w_{2} \\
-\bar{w}_{1} & i a_{2} & w_{2} & z_{2} \\
-\bar{z}_{1} & -\bar{w}_{2} & -i a_{1} & w_{1} \\
-\bar{w}_{2} & -\bar{z}_{2} & -\bar{w}_{1} & -i a_{2}
\end{array}\right) \subset \mathfrak{s} u(4), w_{i}, z_{i} \in \mathbb{C} \text { and } a_{i} \in \mathbb{R} .
$$

The subspace $W$ consists of those matrices with vanishing $a_{i}$ and $z_{i}$. Here is a matrix representation of

$$
W^{\perp}=\left\{\left(\begin{array}{cccc}
0 & 0 & 0 & 0 \\
0 & -i a_{2} & 0 & -z_{2} \\
0 & 0 & 0 & 0 \\
0 & \bar{z}_{2} & 0 & i a_{2}
\end{array}\right),\left(\begin{array}{cc}
i a_{2} & z_{2} \\
-\bar{z}_{2} & -i a_{2}
\end{array}\right)\right\} \in \mathfrak{s} p(2)+\mathfrak{s} p(1) .
$$

By Lemma 2.1.4, the image of the adjoint representation $\rho\left(S p(1)_{1} \times S p(1)_{2}\right)$ on $V=W+W^{\perp}$ is $S O(4)_{3,4}$. Using the invariance principle, we conclude that the normalizer of $S p(1)_{1} \times S p(1)_{2}$ in $G^{s c}=S p(2) \times S p(1)$ is $\left(S p(1)_{1} \times S p(1)_{2}\right) \times \mathcal{Z}(S p(1))$. This proves the first and the second assertion of Proposition 3.1.2. The last assertion follows from a direct computation.

Possibility 2 with $\mathfrak{h}=\mathfrak{s o}(3)$. Recall that there are three sub-cases $(2 \mathrm{a}),(2 \mathrm{~b})$ and (2c). We denote by $S O(3)_{3,3}$ (resp. $S U(2)_{3,4}, S U(2)_{2,4}$ ) the connected Lie subgroup in $S O(4)_{3,4}$ whose Lie algebra is $\mathfrak{s o}(3)_{3,3}\left(\right.$ resp. $\left.\mathfrak{s} u(2)_{3,4}, \mathfrak{s} u(2)_{2,4}\right)$.

From Lemma 2.1.4 we get immediately

Lemma 3.1.3. An embedding $\Pi: s o(3) \rightarrow g l\left(\mathbb{R}^{7}\right)$ can be factored as an embedding $\Pi:$ so $(3) \rightarrow \operatorname{so}(4)_{3,4} \subset g l\left(\mathbb{R}^{7}\right)$, if and only if one of the following three conditions holds.

Case (i). $\Pi$ is a direct sum of two real irreducible representations of dimension 3 and one trivial representation. In this case the image of the induced embedding $\Pi_{*}(\mathfrak{s o}(3))$ is $\mathfrak{s o}(3)_{3,3}$ associated with case (2a).

Case (ii). $\Pi$ is a sum of one real irreducible representation of dimension 4 and one real irreducible representation of dimension 3. In this case the image of the induced embedding $\Pi_{*}(\mathfrak{s o}(3))$ is $\mathfrak{s} u(2)_{3,4}$ associated with case (2b).

Case (iii). $\Pi$ is a sum of a real irreducible representation of dimension 4 and three real representations of dimension 1. In this case the image of the induced embedding $\Pi_{*}(\mathfrak{s} o(3))$ is $\mathfrak{s} u(2)_{0,4}$ associated with case (2c).

Lemma 3.1 .3 implies that $\mathfrak{g}$ cannot contain a commutative ideal of dimension greater than or equal to 4 . Taking into account $\operatorname{dim} \mathfrak{g}=10$, we conclude that $\mathfrak{g}$ 
must be one of the following Lie algebras:

i) $\mathfrak{g}=\mathfrak{s} o(5)$,

ii) $\mathfrak{g}=\mathfrak{s} u(3)+\mathbb{R}^{2}$,

iii) $\mathfrak{g}=3 \mathfrak{s} o(3)+\mathbb{R}$.

Let us denote the element $\operatorname{diag}(1,-1,-1,-1,-1) \in S O(5)$ by $D_{1,4}$. We denote by $p$ the projection from $\operatorname{Spin}(5)$ to $S O(5)$. Then $p^{-1}\left(\mathbb{Z}_{2}\left[D_{1,4}\right]\right)=\mathbb{Z}_{2} \times \mathbb{Z}_{2} \subset$ $\operatorname{Spin}(5)$.

Let $H$ be a Lie subgroup of a Lie group $G$ and $\Gamma$ be a finite subgroup of the normalizer $\mathcal{N}_{G}(H)$. We denote by $H \cdot \Gamma$ the Lie subgroup in $G$ generated by $H$ and $\Gamma$. If the intersection $\Gamma$ with $H$ is the neutral element $e \in G$, and $\Gamma$ is a subgroup of the centralizer $\mathcal{Z}_{G}(H)$, then we also write $H \times \Gamma$ instead of $H \cdot \Gamma$.

Proposition 3.1.4. Suppose that $\hat{G} / H$ admits a $\hat{G}$-invariant $\tilde{G}_{2}$-structure such that their corresponding Lie algebras $(\mathfrak{h} \subset \mathfrak{g})$ are in possibility 2. Then one of the following case happens.

Case (i) with $\hat{G}=\operatorname{Spin}(5)=S p(2)$. Then $H$ is conjugate to one of the following subgroups

- $S p(1) \cdot \Gamma$, where $S p(1)$ is diagonally embedded into $S p(1) \times S p(1) \subset S p(2)=$ $\operatorname{Spin}(5)$ (case (2a)) and $\Gamma \subset p^{-1}\left(\mathbb{Z}_{2}\left[D_{1,4}\right]\right)$. The kernel of the action is $\mathcal{Z}(\operatorname{Spin}(5))=$ $\mathbb{Z}_{2}$.

- $S p(1) \times \Gamma$, where $S p(1)$ is the canonically embedded $S p(1) \subset S p(2)$ (case (2c)) and $\Gamma$ is a finite subgroup in $S p(1)_{2} \subset \mathcal{Z}_{S p(2)}(S p(1))$ described in the proof below. The kernel of the $\hat{G}$-action on $\hat{G} / H$ is $\mathcal{Z}(\hat{G}) \cap \Gamma$.

Case (ii) with $\hat{G}=S U(3) \times T^{2}$. Then $H$ is conjugate to $S U(2) \cdot \Gamma$, where $S U(2)$ corresponds to the irreducible complex representation of $\mathfrak{h}$ into $\mathfrak{s} u(3) \subset \mathfrak{g}$ of dimension 2 (case (2c)) and $\Gamma$ is a finite subgroup of $\mathcal{Z}(S U(3)) \times T^{2}$. The kernel of the $\hat{G}$-action is $\Gamma$.

Case (iii) with $\hat{G}=S p(1) \times S p(1) \times S p(1) \times U(1)$. Then $H$ is $H^{0} \cdot \Gamma$. Here $H^{0}$ is the subgroup $S p(1)$ diagonally embedded in $S p(1) \times S p(1) \times S p(1) \subset \hat{G}$ (case (2a)) and $\Gamma$ is a finite subgroup of $\mathcal{Z}(\hat{G})$. The kernel of the $\hat{G}$-action is $\mathbb{Z}_{2}\left[\mathcal{Z}\left(H^{0}\right)\right] \cdot \Gamma$.

Proof. In case (i) direct computations on Lie algebras show that there are only two possible (up to a conjugation) embeddings $\mathfrak{s o}(3) \rightarrow \mathfrak{s} o(5) \subset g l\left(\mathbb{R}^{5}\right)$ whose irreducible components are of real dimensions 3, 4 respectively. The first one has its adjoint representation on $V$ as a sum of two real irreducible representations of dimension 3 and one trivial representation, so it is case (2a). The corresponding pair of connected Lie groups is $(\operatorname{Spin}(3) \subset \operatorname{Spin}(5))$.

The isotropy representation of the second embedding of $\mathfrak{h}$ into $\mathfrak{s o}(5)$ is a sum of one real irreducible representation of dimension 4 and three real irreducible representations of dimension 1 , so it is case (2c). The corresponding pair of connected Lie groups is $(S p(1) \subset S p(2))$.

We now examine which disconnected Lie subgroup $H$ in $G$ satisfies the condition of case (i). First let us assume that its identity connected component $H^{0}=\operatorname{Spin}(3) \subset \operatorname{Spin}(5)=G$ satisfies the condition of case (ii), associated with possibility (2a). To find the normalizer $\mathcal{N}_{\operatorname{Spin}(5)} \operatorname{Spin}(3)$ we project it into the group $S O(5)$. The normalizer $\mathcal{N}_{S O(5)}(S O(3))$ is $S(O(2) \times O(3))$, according to the invariance principle. The group $S(O(2) \times O(3))$ is generated by $S O(2) \times S O(3)$ and $D_{1,4}$, moreover $S O(2) \cdot \mathbb{Z}_{2}\left[D_{1,4}\right]$ is $\mathcal{Z}_{S O(5)}(S O(3))$. Clearly $\left(A d_{D_{1,4}}\right)_{\mid V}$ belongs to $S O(4)_{3,4}$. Let $H^{\prime}$ be the image of the projection of $H$ on $S O(5)$. Then 
$H^{\prime}=S O(3) \cdot \Gamma$, where $\Gamma \subset\left(S O(2) \cdot \mathbb{Z}_{2}\left[D_{1,4}\right]\right)$. A direct calculation shows that the image of the adjoint action of $\Gamma$ on $V$ preserves the $S O(4)_{3,4}$-invariant subspace $\mathbb{R}^{4} \subset V$, if and only if $\Gamma \subset \mathbb{Z}_{2}\left[D_{1,4}\right]$. (Alternatively we compute that $\mathcal{N}_{S O(4)_{3,4}}\left(S O(3)_{3,3}\right)=S O(3)_{3,3} \cdot \mathbb{Z}_{2}[a]$, where $a$ is the generator of the center $\mathcal{Z}(S O(4))_{3,4}$, which gives us the same conclusion.) A direct computation gives the kernel of the action.

Now we assume that $H^{0}$ satisfies the condition of case (ii), associated with possibility (2c). Using the invariance principle, we observe that the normalizer $\mathcal{N}_{S p(2)}\left(H^{0}\right)$ is $H^{0} \times S p(1)_{2}$, where $S p(1)_{2} \subset \mathcal{Z}_{S p(2)}\left(H^{0}\right)$. Thus $H$ is of the form $H^{0} \times \Gamma$, where $\Gamma$ is a finite subgroup in $S p(1)_{2}$. We observe that the image of the adjoint representation of $H^{0} \times S p(1)_{2}$ on $V$ coincides with the subgroup $S O(4)_{3,4} \subset$ $\tilde{G}_{2} \subset G l(V)$. Thus the adjoint representation of $H$ lifts to an embedding of $\rho(H)$ into $S O(4)_{3,4} \subset \tilde{G}_{2} \subset G l(V)$. This proves Proposition 3.1.4 case (i).

In the second case (ii) the corresponding group $\hat{G}$ is $S U(3) \times T^{2}$. A simple calculation using Lemma 3.1.3 shows that there is only one (up to a conjugation) Lie connected subgroup $H \subset \hat{G}$ such that $\mathfrak{h}=\mathfrak{s o}(3)$, and the image of the isotropy representation of the corresponding connected Lie group $H^{0}$ is a subgroup of $\tilde{G}_{2}$. The group $H^{0}$ is $S U(2) \subset S U(3) \subset \hat{G}$ which corresponds to the irreducible complex representation of $\mathfrak{h}$ of dimension 2. Its isotropy representation is a sum of a real irreducible representation of dimension 4 and three trivial representations, so it corresponds to case $(2 \mathrm{c})$.

To complete our examination of this case (ii) we need only to consider the case of a disconnected subgroup $H$. Suppose that $H$ is a subgroup of $\mathcal{N}_{S U(3) \times T^{2}}\left(H^{0}\right)$ having $H^{0}$ as its identity connected component. According to the invariance principle, $\mathcal{N}_{S U(3) \times T^{2}}\left(H^{0}\right)$ is $S(U(2) \times U(1)) \times T^{2}$. Thus $H$ has the form $H^{0} \cdot \Gamma$, where $\Gamma$ is a finite subgroup of $\mathcal{Z}_{S U(3)}(S U(2)) \times T^{2}$. Since the action of $\Gamma$ on $V$ has at least three trivial components of dimension 1 , we conclude that $\rho(\Gamma)$ is a subgroup of $\rho\left(H^{0}\right)$. Hence $\Gamma \subset \mathcal{Z}(\hat{G}) \times T^{2}$. This proves Proposition 3.1.4 case (ii).

In the last case (iii) the corresponding group $\hat{G}$ is $S p(1) \times S p(1) \times S p(1) \times$ $U(1)$. Using Lemma 3.1.3 we conclude that any connected subgroup $H^{0}$ must be embedded diagonally into $S p(1) \times S p(1) \times S p(1)$. It is easy to check that the isotropy action of $H^{0}$ on $V$ is a sum of two real irreducible representations of dimension 3 and one trivial representation of dimension 1 , so it corresponds to case (2a).

Now we prove that any disconnected subgroup $H \subset \hat{G}$ satisfies the condition of case (iii), if its identity connected component $H^{0}$ does. Let us compute $\mathcal{N}_{\hat{G}}\left(H^{0}\right)$. Since $\operatorname{Aut}\left(H^{0}\right)=\operatorname{Int}\left(H^{0}\right)$, we have $\mathcal{N}_{\hat{G}}\left(H^{0}\right)=H^{0} \cdot \mathcal{Z}_{\hat{G}}\left(H^{0}\right)$. Clearly $\mathcal{Z}_{\hat{G}}\left(H^{0}\right)=$ $H^{0} \cdot \mathcal{Z}(\hat{G})$. Hence the image of $\mathcal{N}_{\hat{G}}\left(H^{0}\right)$ under its isotropy action on $V$ is equal to the image of the isotropy action of $H^{0}$. This completes the proof of Proposition 3.1.4.

Possibility 3 with $\mathfrak{h}=\mathfrak{s o}(3)+\mathbb{R}$. Lemma 3.1.3 implies that $\mathfrak{g}$ cannot contain a commutative ideal of dimension greater than or equal to 5 . Since $\operatorname{dim} \mathfrak{g}=11$, taking into account $\mathfrak{g} \supset \mathfrak{h}$, we conclude that $\mathfrak{g}$ is one of the following Lie algebras

i) $3 \mathfrak{s o}(3)+\mathbb{R}^{2}$,

ii) $\mathfrak{s o}(5)+\mathbb{R}$,

iii) $\mathfrak{s u}(3)+\mathfrak{s o}(3)$,

iv) $\mathfrak{s} u(3)+\mathbb{R}^{3}$. 
We exclude the last case (iv), since by Lemma 3.1 .3 the adjoint representation of $\mathfrak{h}$ on $V$ restricted to $\mathfrak{s o}(3) \subset \mathfrak{h}$ has no irreducible component of dimension 5 , and if this representation has an irreducible component of real dimension 4 , the other irreducible subspace has real dimension 3.

Suppose that $H_{1}$ and $H_{2}$ are connected Lie subgroups in a connected Lie group $G$ such that their Lie algebras $\mathfrak{h}_{1}$ and $\mathfrak{h}_{2}$ satisfy the condition $\left[\mathfrak{h}_{1}, \mathfrak{h}_{2}\right]=0$. Then we denote by $H_{1} \cdot H_{2}$ the connected Lie subgroup in $G$ whose Lie algebra is the direct sum $\mathfrak{h}_{1}+\mathfrak{h}_{2}$. From Lemma 2.1.4 we get immediately

Lemma 3.1.5. In group $S O(4)_{3,4}$ there is no subgroup of the form $S O(3) \cdot U(1)$. A subgroup $S U(2) \cdot U(1) \subset G l\left(\mathbb{R}^{7}\right)$ corresponding to a representation $\Pi: \mathfrak{s} u(2)+\mathbb{R} \rightarrow$ $\mathfrak{g l}\left(\mathbb{R}^{7}\right)$ can be seen as a subgroup of $S O(4)_{3,4} \subset G l\left(\mathbb{R}^{7}\right)$, if and only if one of the following two conditions (i) and (ii) is fulfilled.

(i) $\Pi$ is a sum of one real irreducible component of dimension 4, corresponding to the highest weight $(1,1)$ on its Cartan subalgebra, and one real irreducible component of dimension 2, corresponding to the highest weight $(0,1)$ on its Cartan subalgebra, and one trivial component of dimension 1 (so $\bar{\rho}(\mathfrak{h})$ is in situation (3b)).

(ii) $\Pi$ is a sum of one real irreducible component of dimension 4, corresponding to the highest weight $(1,1)$ on its Cartan subalgebra, and one real irreducible component of dimension 3, corresponding to the highest weight $(2,0)$ on its Cartan subalgebra (so $\bar{\rho}(\mathfrak{h})$ is in situation (3a)).

Using Lemma 3.1.5 (or Lemma 3.1.3) we also exclude the first case (i) of possibility 3 by looking at all possible embeddings of the summand $\mathfrak{h} \subset \mathfrak{s o}(3)$ into $\mathfrak{g}=2 \mathfrak{s o}(3)+\mathbb{R}^{2}$. It remains to consider cases (ii) and (iii).

Proposition 3.1.6. Suppose that $\hat{G} / H$ admits a $\hat{G}$-invariant $\tilde{G}_{2}$-structure such that their corresponding Lie algebras $(\mathfrak{h} \subset \mathfrak{g})$ are in possibility 3. Then one of the following cases happens. (A detailed description of $H$ will be given in the proof.)

In case (ii) with $\hat{G}=S p(2) \times U(1)_{2}$, the Lie subgroup $H$ is $S U(2) \cdot U(1)_{k, l} \cdot \Gamma$ with $k \neq 0,(k, l)=1$, and $\Gamma$ is a finite subgroup of $U(1)_{2}$. The kernel of the action is $\mathbb{Z}_{2}[\mathcal{Z}(S p(2))] \times \Gamma$.

In case (iii) with $\hat{G}=S U(3) \times S U(2)$, the Lie subgroup $H$ is one of the following forms.

- $H=S U(2)_{2,0} \cdot U(1)_{k, l} \cdot \Gamma$, where $\Gamma$ is a finite subgroup in $\mathcal{Z}(\hat{G})$, (so $\bar{\rho}(\mathfrak{h})$ is in case (3b)), moreover $k l \neq 0$. We have $\mathcal{Z}(\hat{G}) \cap H^{0}=I d$, if $(2 k-3 l)(4 k-3 l) \neq 0$. In general $\mathcal{Z}(\hat{G}) \cap H$ can be any subgroup of $\mathcal{Z}(\hat{G})=\mathbb{Z}_{3} \times \mathbb{Z}_{2}$ depending on $\Gamma$ and $k, l$.

- $H=S U(2)_{2,3} \cdot U(1)_{1,0} \cdot \Gamma$, where $\Gamma \subset \mathcal{Z}(\hat{G})$, (so $\bar{\rho}(\mathfrak{h})$ is in case (3a)). The kernel of the $\hat{G}$-action is $\left.\mathbb{Z}_{2}\left[\mathcal{Z}(\hat{G}) \cap H^{0}\right)\right] \cdot \Gamma$.

Proof. Let us consider case (ii) with $\mathfrak{g}=\mathfrak{s} o(5)+\mathbb{R}$. We can assume that the projection $\Pi_{1}(\mathbb{R})$ of the summand $\mathbb{R} \subset \mathfrak{h}$ on $\mathfrak{s o}(5)$ is nonzero, otherwise the kernel of the isotropy action of $\mathfrak{h}$ contains $\mathbb{R}$, and the action of $\bar{\rho}(\mathfrak{h})$ is not faithful.

A direct computation shows that the embedding of $\mathfrak{s o}(3)$ to $\mathfrak{s o}(5)$ is associated with a real irreducible representation of $\mathfrak{s o}(3)$ of dimension 4 (complex dimension $2)$, and the projection $\Pi_{1}(\mathfrak{h})$ is the Lie algebra of the centralizer $\mathcal{Z}_{\mathfrak{s} o(5)}(\mathfrak{s o}(3))$. A subgroup $S U(2) \times U(1) \subset S p(2) \times U(1)_{2}$ having this Lie algebra is determined by 2 integers $(k, l)$ which are the coordinates of the component $U(1)$ w.r.t. $U(1)_{1} \subset$ $\mathcal{Z}_{S p(2)}(S U(2))$ and $U(1)_{2}$. We denote this subgroup by $S U(2) \cdot U(1)_{k, l}$. By our 
condition $k \neq 0$ and $(k, l)=1$. We check easily that the associated isotropy representation of $S U(2) \cdot U(1)_{k, l} \subset S p(2) \times U(1)_{2}$ corresponds to case (i) in Lemma 3.1 .5 .

Now let us find all Lie subgroups $H$ in $\hat{G}$ satisfying the condition of Proposition 3.1.6, case (ii). By our consideration above it follows that the identity connected component $H^{0}$ of $H$ is embedded in $S p(2) \times U(1)_{2}$ as $S U(2) \cdot U(1)_{k, l}$. Clearly $U(1)_{2} \subset \mathcal{N}_{\hat{G}}\left(H^{0}\right)$. Using the invariance principle we conclude that $\mathcal{N}_{\hat{G}}\left(H^{0}\right)=$ $H^{0} \times U(1)_{2}$. This proves the first assertion of Proposition 3.1.6, case (ii). The second assertion follows by a direct calculation.

Now let us consider case (iii) with $\mathfrak{g}=\mathfrak{s} u(3)+\mathfrak{s} o(3)$. Denote by $\Pi_{1}$ the projection of $\mathfrak{h}$ on the summand $\mathfrak{s} u(3) \subset \mathfrak{g}$ and by $\Pi_{2}$ the projection of $\mathfrak{h}$ on the summand $\mathfrak{s o}(3) \subset \mathfrak{g}$. Using Lemma 3.1 .5 we conclude that $\Pi_{1}(\mathbb{R})$ is nonzero, otherwise the restriction of the isotropy action to the summand $\mathbb{R} \subset \mathfrak{h}$ would have at least 5 trivial components. Repeating this argument, we conclude that $\Pi_{1}(\mathfrak{s o} o(3))$ is also nonzero. Clearly the embedding of $\Pi_{1}(\mathfrak{s} o(3))$ into $\mathfrak{s} u(3) \subset \mathfrak{g l}\left(\mathbb{C}^{3}\right)$ must correspond to its complex irreducible representation of complex dimension 2 , because its image commutes with $\Pi_{1}(\mathbb{R})$. Hence the projection $\Pi_{1}(\mathfrak{h})$ is defined uniquely up to automorphisms of $\mathfrak{s} u(3)$. Hence the embedding of the component $U(1)$ into $S U(3) \times S U(2)=G^{s c}$ is characterized by two integers $(k, l)$ which are the coordinates of $U(1)$ w.r.t. $U(1)_{1}$ and $U(1)_{2}$, where $U(1)_{1}=\mathcal{Z}_{S U(3)}(S U(2))$ and $U(1)_{2}$ being a maximal torus of $S U(2)$. Further we observe that there are two possible sub-cases.

If $\Pi_{2}(\mathfrak{s o}(3))$ is empty, then $k \neq 0$ and $l \neq 0$. Denote by $S U(2)_{2,0} \cdot U(1)_{k, l}$ the connected Lie subgroup of $S U(3) \times S U(2)$ having Lie algebra $\mathfrak{h}$ with this property. Its isotropy representation corresponds to case (3b) in Lemma 3.1.5.(i).

If $\Pi_{2}(\mathfrak{s o}(3))$ is not empty, then $l=0$, and hence $k=1$. Denote by $S U(2)_{2,3}$. $U(1)_{1,0}$ the Lie subgroup of $S U(3) \times S U(2)$ having Lie algebra $\mathfrak{h}$ with this property. Its isotropy representation corresponds to case (3a), see also Lemma 3.1.5. (ii).

Now we consider disconnected Lie subgroups $H$ whose Lie algebra $\mathfrak{h}$ is in case (iii), the first sub-case (3b). Denote by the same $\Pi_{i}$ the lift of $\Pi_{i}$ from $\mathfrak{g}$ to $\hat{G}$. Since $k \cdot l \neq 0$, we have

$$
\Pi_{2}\left[\mathcal{N}_{\hat{G}}\left(S U(2)_{2,0} \cdot U(1)_{k, l}\right)\right] \subset \mathcal{N}_{S U(2)} \Pi_{2}\left(U(1)_{k, l}\right)=\Pi_{2}\left(U(1)_{k, l}\right) \cdot \mathbb{Z}_{2}\left[A_{(12)}\right] .
$$

Here $A_{(12)}=\left(\begin{array}{cc}0 & \sqrt{-1} \\ \sqrt{-1} & 0\end{array}\right) \in S U(2)$.

But $A d_{A_{(12)}}$ maps $U(1)_{k, l}$ to $U(1)_{k,-l}$. Now it is easy to see that $\mathcal{N}_{\hat{G}}\left(S U(2)_{2,0}\right.$. $\left.U(1)_{k, l}\right)=S U(2)_{2,0} \cdot U(1)_{k, l} \cdot U(1) \cdot \mathcal{Z}(\hat{G})$, where $U(1) \subset S U(2) \subset \hat{G}$.

Let $x \in H \cap U(1)$. Since $x$ commutes with $H^{0}$, if $A d_{x}$ belongs to $S O(4)_{3,4}$, the image $A d_{x}$ must belong to $A d_{U(1)_{k, l}}$. Hence $x \in \mathcal{Z}(\hat{G})$. This proves $H \subset H^{0} \cdot \mathcal{Z}(\hat{G})$. A direct calculation gives the kernel of the $\hat{G}$-action in this case.

In the next sub-case (3a), using the invariance principle, we conclude that $\mathcal{N}_{\hat{G}}\left(S U(2)_{2,3} \cdot U(1)_{1,0}\right)=S U(2)_{2,3} \cdot U(1)_{1,0} \cdot \mathcal{Z}(\hat{G})$. A direct computation completes the proof of Proposition 3.1.6.

Possibility \& with $\mathfrak{h}=\mathbb{R}^{2}$. If $r k \mathfrak{g} \geq 4$, then the dimension of the fixed-point of the action of $\rho(H)$ on $V$ is at least 2 which does not agree with the action of the maximal torus of $S O(4)_{3,4}$ on $\mathbb{R}^{7}$. Thus $\mathfrak{g}$ must be one of the following Lie algebras 
i) $\mathfrak{s o}(3)+\mathfrak{s} o(3)+\mathfrak{s} o(3)$,

ii) $\mathfrak{s} u(3)+\mathbb{R}$.

In case (ii) instead of working with $\hat{G}=S U(3) \times U(1)$ it is more convenient to work with $G=U(3)=\hat{G} / D_{3}$, where $D_{3}=\left\{\left(g, g^{-1}\right) \mid g=\operatorname{diag}\left(e^{\left(\frac{\sqrt{-1} 2 k \pi}{3}\right.}, e^{\frac{\sqrt{-1} 2 k \pi}{3}}, e^{\frac{\sqrt{-1} 2 k \pi}{3}}\right), k=\right.$ $1,2,3\}$. We note that there is a 1-1 correspondence between connected Lie subgroups $H$ in $\hat{G}$ and connected Lie subgroups $H^{\prime}$ in $U(3)$ having the same Lie algebra $\mathfrak{h} \subset \mathfrak{g}=\mathfrak{s} u(3)+\mathbb{R}$. Furthermore, $\mathcal{N}_{\hat{G}}(H)=\pi^{-1}\left(\mathcal{N}_{U(3)}\left(H^{\prime}\right)\right)$, where $\pi: \hat{G} \rightarrow U(3)$ is the natural projection. Thus it suffices to work with $G=U(3)$. As we will see below, $\mathcal{N}_{G}\left(H^{\prime}\right)$ is generated by $H^{\prime}$ and a subgroup $\Gamma \subset \mathcal{Z}(G)$. Hence, to get a full list of a classification in case (ii), working with $G=U(3)$ instead of $\hat{G}$, we need examine only one extra possibility, if the corresponding connected Lie subgroup $H \subset \hat{G}$ contains $D_{3}$.

Proposition 3.1.7. In case (i) with $\hat{G}=S U(2) \times S U(2) \times S U(2)$, the Lie subgroup $H$ is of the form $U(1)_{0,1,-1} \cdot U(1)_{1,0,-1} \cdot \Gamma$, where $\Gamma \subset \mathcal{Z}(\hat{G}) \times \mathbb{Z}_{2}\left[\left(A_{(12)}, A_{(12)}, A_{(12)}\right)\right]$. The kernel of the $\hat{G}$-action is $\mathbb{Z}_{2}[-I d,-I d, I d] \cdot(\Gamma \cap \mathcal{Z}(\hat{G}))$.

In case (ii) with $G=U(3)$, the Lie subgroup $H$ is of the form of $U(1)_{k, k, k+1}$. $U(1)_{m, m+1, m+1} \cdot \Gamma$, where $\Gamma \subset \mathcal{Z}(G)$. The kernel of the $G$-action is $\Gamma$. $A$ detailed description of $H$ will be given in the proof below.

Proof. Let us fix a subgroup $S O(2)_{2,2} \subset S O(3)_{3,3} \subset S O(4)_{3,4}$. We can choose a subgroup $U(1)_{0,4} \subset S U(2)_{0,4} \subset S O(4)_{3,4}$ such that these subgroups are generators of a maximal torus of $S O(4)_{3,4}$. Any subgroup $U(1)$ in this torus shall be denoted by $U(1)_{p, q}$ with respect to this lattice.

In case (i) let us fix a maximal torus $U(1)_{1} \times U(1)_{2} \times U(1)_{3}$ of $\hat{G}=S U(2)_{1} \times$ $S U(2)_{2} \times S U(2)_{3}$ such that $U(1)_{i} \subset S U(2)_{i}$. Let $T^{2}$ be a torus in $\hat{G}$ such that $\rho\left(T^{2}\right) \subset S O(4)_{3,4}$. W.l.g. we can assume that $\rho\left(T^{2}\right)=U(1)_{0,4} \cdot S O(2)_{2,2}$. Let $U(1)_{k, l, m}$ be the preimage $\rho^{-1}\left(U(1)_{p, q}\right)$, where $(k, l, m)$ are the coordinates with respect to $U(1)_{i}$. The weights of the adjoint action of $\rho^{-1}\left(U(1)_{p, q}\right)$ on $V$ are ( $1, \exp \pm 2 k \sqrt{-1} \theta, \exp \pm 2 l \sqrt{-1} \theta, \exp \pm 2 m \sqrt{-1} \theta$ ) which must coincide with the weights of the representation of $U(1)_{p, q}$ on $\mathbb{R}^{7}$ which are $(1, \exp \pm \sqrt{-1} p \theta, \exp \pm \sqrt{-1} q \theta$, $\exp \mp \sqrt{-1}(p+q) \theta)$. Taking into account that the isotropy action of $U(1)_{i}$ on $V_{i} \subset \mathfrak{s} u(2)_{i}$ is a double covering, we conclude that $k= \pm p, l= \pm q, m=\mp(p+q)$. Each choice of the sign of the weights of the action of the torus on $V^{7}$ corresponds to a different solution of the coordinates $(k, l, m)$ of $T^{2}$. Observing that $T^{2}$ is invariant under the inverse map $x \mapsto x^{-1}$, we have actually only four different solutions for the coordinates $(k, l, m)$. Using the permutations between $S U(2)_{i}$, we get only three different solutions for $T^{2}$ :

$T_{1}^{2}=U(1)_{0,1,-1} \times U(1)_{1,0,-1}, T_{2}^{2}=U(1)_{0,1,1} \times U(1)_{1,0,1}, T_{3}^{2}=U(1)_{0,-1,1} \times U(1)_{1,0,1}$.

It is easy to see that $T_{2}^{2}$ and $T_{3}^{2}$ are equivalent up to automorphism of $\hat{G}$. Since we can change the orientation of each $U(1)_{i} \subset S U(2)_{i}$, the tori $T_{1}^{2}$ and $T_{2}^{2}$ are equivalent. Thus up to conjugation by automorphism of $\hat{G}$, there is only one choice of $T^{2}$ satisfying our condition.

To complete the examination of case (i) we need to find all disconnected Lie subgroup $H$ whose identity connected Lie component $H^{0}$ is the torus $U(1)_{0,1,-1}$. $U(1)_{1,0,-1}$. Clearly $T^{3}=U(1)_{1} \times U(1)_{2} \times U(1)_{3} \subset \mathcal{N}_{\hat{G}}\left(H^{0}\right)$. Considering the 
projection of $\mathcal{N}_{\hat{G}}\left(H^{0}\right)$ on each factor $S U(2)_{i}$ we conclude that $\mathcal{N}_{\hat{G}}\left(H^{0}\right) \subset T^{3}$. $\left(\mathbb{Z}_{2}\left[A_{(12)}\right]\right)^{3}$.

A direct calculation shows that $\mathcal{N}_{\hat{G}}\left(H^{0}\right)=T^{3} \cdot \mathbb{Z}_{2}\left[\left(A_{(12)}, A_{(12)}, A_{(12)}\right)\right]$. Hence $H=H^{0} \cdot \Gamma$, where $\Gamma$ is a finite subgroup in $T^{3} \cdot \mathbb{Z}_{2}\left[\left(A_{(12)}, A_{(12)}, A_{(12)}\right)\right]$. Further we note that the element $A d_{\left(A_{(12)}, A_{(12)}, A_{(12)}\right)}$ in $S O\left(V^{7}\right)$ is $D_{7}=\operatorname{diag}(-1,1,-1,1-$ $1,1,-1)$, which belongs to $S O(4)_{3,4}$.

Clearly the element $A d_{x}$ in $S O\left(V^{7}\right)$, where $x \in T^{3}$, belongs to $S O(4)_{3,4}$, if and only if $x \in H^{0} \cdot \mathcal{Z}(G)$. Thus the image $\rho\left(H^{0} \cdot \Gamma\right)$ belongs to $S O(4)_{3,4}$, if and only if $\Gamma \subset \mathcal{Z}(G) \cdot \mathbb{Z}_{2}\left[\left(A_{12}, A_{(12)}, A_{(12)}\right)\right]$. A direct computation yields the second statement of Proposition 3.1.7.

It remains to consider case (ii) with the corresponding group $G=U(3)$. Now we use the notations $U(1)_{1}, U(1)_{2}, U(1)_{3}$ for the generators of the maximal torus of $U(3)$. Suppose that there is $T^{2} \subset U(1)_{1} \times U(1)_{2} \times U(1)_{3}$ such that $\rho\left(T^{2}\right)=$ $U(1)_{0,4} \cdot S O(2)_{2,2} \subset S O(4)_{3,4}$. The weights of the isotropy action of $U(1)_{k, l, m}$ is $(1, \exp \pm \sqrt{-1}(k-l), \exp \pm(l-m), \exp \sqrt{-1}(k-m))$ and the weights of the representation of $U(1)_{p, q}$ are $(\exp \pm p, \exp \pm q, \exp \mp(p+q))$. Thus $T^{2}$ must be $U(1)_{k, k, k+1} \cdot U(1)_{m, m+1, m+1}$ or $U(1)_{k, k, k-1} \cdot U(1)_{m, m-1, m-1}$. These two families of solutions are actually mirror identical.

Now let us find all disconnected Lie group $H$ whose identity component $H^{0}$ is conjugate to the torus $T_{k, m}^{2}=U(1)_{k, k, k+1} \cdot U(1)_{m, m+1, m+1}$. Since $\mathfrak{h}$ contains a regular element, it follows that the identity connected component of $\mathcal{Z}_{\hat{G}}\left(H^{0}\right)$ is a torus $T^{3}$. Denote by $l T^{3}$ the Lie algebra of $T^{3}$. Using the invariance principle applying to $W_{1}^{\perp}=l T^{3} \subset \mathfrak{g}$, we conclude that $\mathcal{N}_{G}\left(H^{0}\right)$ leaves $T^{3}$ invariantly. Hence $\mathcal{N}_{\hat{G}}\left(H^{0}\right)$ is a subgroup of $\mathcal{N}_{\hat{G}}\left(T^{3}\right)=T^{3} \cdot \Sigma_{3}$, where $\Sigma_{3}$ is the Weyl group generated by two elements of order 3 and of order 2 in $S U(3)$.

Since $\left(A d_{\Sigma_{3}}\right)_{\mid \mathfrak{g}} \subset S O(\mathfrak{g})$, an element $x \in \Sigma_{3}$ belongs to the normalizer $\mathcal{N}_{G}\left(H^{0}\right)$, if and only if it leaves the orthogonal complement $\langle(-(m+1),(m-k), k)\rangle_{\mathbb{R}}$ of $\mathfrak{h}$ in $l T^{3}=\mathbb{R}^{3}$ invariantly. The generators of $\Sigma_{3}$ are

$$
A_{(123)}=\left(\begin{array}{lll}
0 & 0 & 1 \\
1 & 0 & 0 \\
0 & 1 & 0
\end{array}\right) \text { and } B_{(23)}=\left(\begin{array}{ccc}
-1 & 0 & 0 \\
0 & 0 & 1 \\
0 & 1 & 0
\end{array}\right) \text {. }
$$

They act on $T^{3}$ by permuting coordinates $k, l, m$. We conclude that $\mathcal{N}_{\hat{G}}\left(H^{0}\right)=$ $T^{3}$, if $T_{k, m}^{2}$ is regular, i.e. if all three coordinates $-(m+1),(m-k), k$ are mutually different. If $T_{k, m}$ is singular, $\mathcal{N}_{\hat{G}}\left(H^{0}\right)=T^{3} \cdot \Gamma_{0}$, where $\Gamma_{0} \subset \Sigma_{3}$ and $A d_{\Gamma_{0}}$ permutes two equal coordinates of $(-(m+1),(m-k), k)$. Arguing as in case (i), we conclude that for regular tori $T_{k, m}^{2}$ we have $H=H^{0} \cdot \Gamma$, where $\Gamma \subset \mathcal{Z}(G)$. In this case $\mathcal{Z}(G) \cap H=\Gamma$. For a singular torus $T_{k, m}^{2}=H^{0}$ we need also to consider the case, when $H$ contains an element of $\Sigma_{3}$. A direct computation shows that the action $A d_{x}, x \in \Sigma_{3}$, permuting two coordinates of $T^{3}$ acts on the invariant subspace $\mathbb{R}^{3} \subset \mathbb{R}^{7}$ as $(1,1,-1)$, hence it does not belong to $S O(4)_{3,4}$. Thus this case cannot happen. This completes our proof.

Possibility 5 with $\mathfrak{h}=\mathbb{R}$. Clearly $r k \mathfrak{g} \leq 5$, since the action of any group $U(1) \subset$ $\tilde{G}_{2}$ on $\mathbb{R}^{7}$ is non-trivial. Since $\operatorname{dim} \mathfrak{g}=8$, we conclude that $\mathfrak{g}$ is one of the following Lie algebras:

i) $2 \mathfrak{s o}(3)+\mathbb{R}^{2}$,

ii) $\mathfrak{s} u(3)$. 
The Lie group $\hat{G}$ with Lie algebra $2 \mathfrak{s o}(3)+\mathbb{R}^{2}$ is isomorphic to $S U(2) \times S U(2) \times T^{2}$. By the same argument as in our consideration of possibility 4 , case (ii), we can work equally with the group $U(2) \times U(2)$ instead with $\hat{G}$. To distinguish the isomorphic factors $U(2)$ in this decomposition of $G$, we denote them by $U(2)_{1}$ and $U(2)_{2}$.

Proposition 3.1.8. In case (i) with $G=U(2)_{1} \times U(2)_{2}$, the Lie subgroup $H$ is $U(1)_{k, k+1, l, l+1} \cdot \Gamma$, where $\Gamma$ is a finite subgroup of $\mathcal{Z}(G)=U(1)_{1} \times U(1)_{2}$. The kernel of this action is $\Gamma$.

In case (ii) with $\hat{G}=S U(3)$ the Lie subgroup $H$ is $U(1)_{k, l, m} \times \Gamma$, where $(k, l)=1$, and $\Gamma$ is any finite subgroup of the maximal torus $T^{2} \subset S U(3)$. If $k=l=1$, then $H$ can also take the form $U(1)_{1,1,-2} \cdot \Gamma$, where $\Gamma$ is a finite subgroup in $S U(2)$. If $k \neq l$, the kernel of the $\hat{G}$-action is either Id or $\mathcal{Z}(\hat{G})$, depending on $\Gamma$. If $k=l=1$, the kernel of the $\hat{G}$-action is $\mathcal{Z}(\hat{G})=\mathbb{Z}_{3}$.

$A$ detailed description of $H$ will be given in the proof.

Proof. Let us consider case (i) with $G=U(2)_{1} \times U(2)_{2}$. Any embedding $U(1)=$ $\exp \mathfrak{h}$ into $G$ is characterized by a quadruple of integers $\left(k_{1}, k_{2}, l_{1}, l_{2}\right)$ which are coordinates of $U(1)$ in $U(1)_{11} \times U(1)_{12} \times U(1)_{21} \times U(1)_{22}$, where $U(1)_{i j} \times U(1)_{i i}$ is a maximal torus of $U(2)_{i}$. We denote by $U(1)_{k_{1}, k_{2}, l_{1}, l_{2}}$ this subgroup exp $\mathfrak{h}$. The isotropy action of $U(1)_{k_{1}, k_{2}, l_{1}, l_{2}}$ with parameter $\theta$ has weights (exp $\pm \sqrt{-1}\left(k_{1}-\right.$ $\left.\left.k_{2}\right) \theta, \exp \pm \sqrt{-1}\left(l_{1}-l_{2}\right) \theta, 1,1,1\right)$. Note that $\rho\left(U(1)_{k_{1}, k_{2}, l_{1}, l_{2}}\right)$ can be written as $U(1)_{p, q}$ as in the proof of Proposition 3.1.7. case (i). Since the weights of the representation of $U(1)_{p, q}$ on $\mathbb{R}^{7}$ are $(1, \exp \pm \sqrt{-1} p \theta, \exp \pm \sqrt{-1} q \theta, \exp \mp \sqrt{-1}(p+$ $q) \theta$ ) coincide with the weights of the isotropy action of $U(1)_{k_{1}, k_{2}, l_{1}, l_{2}}$, we conclude that $U(1)_{p, q}$ must be either $U(1)_{0,4}$ or $S O(2)_{2,2}$ (cf. with the proof of Proposition 3.1.7. case (i)). So $k_{1}-k_{2}= \pm 1$ and $l_{1}-l_{2}= \pm 1$. Up to automorphism of $G$ all these solution subgroups are equivalent, so we will take a representative $U(1)_{k, k+1, l, l+1}$ of these solutions.

We compute $\mathcal{N}_{G}\left(U(1)_{k, k+1, l, l+1}\right)$ easily, by using the projection of this subgroup on each component $U(2)_{i} \subset G$. Knowing $\mathcal{N}_{S U(2)}(U(1))=U(1) \cdot \mathbb{Z}_{2}\left[A_{(12)}\right]$ we conclude that $\mathcal{N}_{G}\left(U(1)_{k, k+1, l, l+1}\right)=T^{4}$, if $(k+1)^{2}+(l+1)^{2} \neq 0$. Otherwise $\mathcal{N}_{G}\left(U(1)_{-1,1,-1,1}\right)=T^{4} \cdot \mathbb{Z}_{2}\left[\left(A_{(12)}, A_{(12)}\right)\right]$.

In the first case $H=U(1)_{k, k+1, l, l+1} \times \Gamma$, where $\Gamma$ is a finite subgroup of $T^{4}$. Since the isotropy action of $\left(\exp \sqrt{-1} \theta_{1}, \exp \sqrt{-1} \theta_{2}, \exp \sqrt{-1} \tau_{1}, \exp \sqrt{-1} \tau_{2}\right)$ acts on the fixed-point subspace $\mathbb{R}^{3} \subset \mathbb{R}^{7}$ of $\rho\left(H^{0}\right)$ as the identity, we conclude that $\left(A d_{\Gamma}\right)_{\mid V} \subset A d_{U(1)_{k, k+1, l, l+1}}$, hence $\Gamma \in H^{0} \cdot \mathcal{Z}(G)$.

In case $k=1=l$, a direct computation shows that the action of $A d_{\left(A_{(12)}, A_{(12)}\right)}$ changes orientation of $V^{7}$. Thus the examination of this case can be done as in the previous case with $k \neq l$. This proves the first assertion of Proposition 3.1.8. The second assertion follows by direct computation.

Now let us consider case (ii). An embedding $\exp \mathfrak{h}=U(1) \rightarrow T^{2} \subset S U(3)=G^{s c}$ can be characterized by a triple $(k, l, m)$ with $k+l+m=0$ and $(k, l)=1$. We denote this subgroup by $U(1)_{k, l, m}$. The weights of the isotropy action of $\mathfrak{h}$ on $V$ are $(0, \pm \sqrt{-1}(k-l), \pm \sqrt{-1}(l-m), \pm \sqrt{-1}(m-k))$. The group $\rho(U(1))$ can be embedded into $S O(4)_{3,4}$ by setting the coordinates $p, q$ of this subgroup $\rho(U(1))$ in the maximal torus $T^{2}$ of $S O(4)_{3,4}$ whose basis is subgroups $U(1)_{0,4} \subset S U(2)_{0,4}$ and $S O(2)_{2,2} \subset S O(3)_{3,3}$ as above. Since the weights of the action of $U(1)_{p, q}$ on $\mathbb{R}^{7}$ are $1, \exp \pm \sqrt{-1} p \theta, \exp \pm \sqrt{-1}(-p+q) \theta, \exp \mp \sqrt{-1} q \theta$, we have $p=(k-l),-p+q=$ $(l-m), q=k-m$. If $k \neq l$, then $\operatorname{ker} \rho_{U(1)_{1,1,-2}}=\mathbb{Z}_{3}$. 
To compute the normalizer $\mathcal{N}_{S U(3)}\left(U(1)_{k, l, m}\right)$, as in the previous case, we observe that the connected component of $\mathcal{Z}_{S U(3)}\left(U(1)_{k, l, m}\right)$ is $T^{2}$. Applying the invariance principle, we conclude that $\mathcal{N}_{S U(3)}\left(U(1)_{k, l, m}\right)$ leaves the torus $T^{2}$ invariantly. Hence $\mathcal{N}_{S U(3)}\left(U(1)_{k, l, m}\right)$ is a subgroup of the normalizer $\mathcal{N}_{S U(3)}\left(T^{2}\right)=T^{2} \cdot \Sigma_{3}$. Arguing as in possibility 4 , case (ii), we conclude that an element $x \in \Sigma_{3}$ normalizes $U(1)_{k, l, m}$, only if $x=I d$, because $(k, l, m)$ is regular. Thus $\mathcal{N}_{S U(3)}\left(U(1)_{k, l, m}\right)=T^{2}$, for $(k, l)=1$ and $k \neq l$. It is known that $\mathcal{N}_{S U(3)} U(1)_{1,1,-2}=S U(2) \cdot U(1)_{1,1,-2}$.

Now let us consider a disconnected Lie subgroup $H$ whose identity component $H^{0}$ is $U(1)_{k, l, m}$. Clearly $H=H^{0} \times \Gamma$, where $\Gamma$ is a subgroup of the maximal torus $T^{2} \subset S U(3)$. The same argument as in the previous case implies that the image of $A d_{T^{2}}$ is the maximal torus of $S O(4)_{3,4}$. This proves the third assertion of Proposition 3.1.8 Applying Lemma 3.1.5. ii we prove the assertion for the case $k=l=1$. A direct computation of $\mathcal{Z}(G) \cap H$ completes the proof of Proposition 3.1 .8 .

Possibility 6 with $\mathfrak{h}=0$. In this case $H$ is a finite subgroup of a compact group $G$ dimension 7 . Thus $\hat{G}$ is one of the following cases:

6i) $T^{7}$,

6ii) $S U(2) \times T^{4}$,

6iii) $S U(2) \times S U(2) \times U(1)$.

Clearly any group $G$ listed above admits a $G$-invariant 3 -form of $\tilde{G}_{2}$-type. Since $T^{7}$ is commutative, wee need only to verify in case (6ii) (resp. case (6iii)), whether there is a finite non-central subgroup $H \subset G$ such that $\rho(H) \subset S O(3)$ (resp. $\rho(H) \subset S O(3) \times S O(3))$ is a subgroup of $S O(4)_{3,4} \subset G_{2}$. In case (6ii) the action of any element $e \in \rho(H)$ on $\mathbb{R}^{7}$ leaves a subspace $\mathbb{R}^{5}$ invariant. On the other hand, any element $e \neq I d \in S O(4)_{3,4}$ is conjugate to an element in $T^{2} \subset S U(3) \subset$ $S O(6) \subset S O(7)$, which cannot have its fixed point subspace in $\mathbb{R}^{7}$ of dimension greater than 3. Thus $\rho(H)$ consists only of the identity. In case (6iii), let $S O(4)_{3,4}$ be a maximal compact subgroup in $\tilde{G}_{2}$ containing $\rho(H)$, whose existence follows from [14, Theorem 2.1, p. 256] (see also Lemma 2.1.3). We note that $\rho(H)$ is a subgroup of $S O(3) \times S O(3)$ as well as a subgroup of $G l\left(\mathbb{R}^{6}\right) \cap G_{2}=S U(3)$, (this is a consequence of the transitivity of the $G_{2}$-action on $S^{6} \subset \mathbb{R}^{7}$ [3], or see [8, §2] for an alternative argument), taking into account that $S O(4)_{3,4}$ is also a subgroup of $G_{2}$ by Lemma 2.2.2. Let $V_{1}=\mathbb{R}^{3}$ and $V_{2}=\mathbb{R}^{3}$ be invariant subspaces of $\rho(H)$ and $J$ be the complex structure on $\mathbb{R}^{6}$. There are two possibilities: either $V_{2}=J V_{1}$, or $J V_{1} \cap V_{1}=\mathbb{R}_{1}^{2}$ and $J V_{2} \cap V_{2}=\mathbb{R}_{2}^{2}$. In the first possibility $\rho(H)$ is a subgroup of $\left(S O\left(V_{1}\right) \times S O\left(V_{2}\right)\right) \cap S U(3)=S O(3)_{3,3}$. In the second possibility $\rho(H)$ is a cyclic subgroup of the form $\left(x, x^{-1}\right) \in S O(3) \times S O(3)$. Clearly these subgroups belong to $S O(4)_{3,4}$. Thus we get

Proposition 3.1.9. i) Let $H$ be a finite subgroup of $T^{7}$. The action of $T^{7}$ on $T^{7} / H$ is effective, iff $H=\{e\}$.

ii) Let $H$ be a finite subgroup of a compact Lie group $G=S U(2) \times T^{4}$. The quotient space $G / H$ admits a $G$-invariant 3-form of $\tilde{G}_{2}$-type, if and only if $\rho(H)$ is central. iii) Let $H$ be a finite not central subgroup of a compact Lie group $G=S U(2) \times$ $S U(2) \times U(1)$. The quotient space $G / H$ admits a $G$-invariant 3 -form of $\tilde{G}_{2}$ type, if and only if $\rho(H)$ is a subgroup of $S O(3)_{3,3}$ or a cyclic group of the form $\left(x, x^{-1}\right) \in S O(3) \times S O(3)$. 
3.2. Classification theorem. In this subsection we summarize our computation in the previous subsection in the following Theorem 3.2.1, taking into account our remarks before Propositions 3.1.7 and 3.1.8. We also provide a formula to compute the dimension of the space of all invariant $\tilde{G}_{2}$-structures on a given manifold $G / H$, see Remark 3.2.2, d.

Theorem 3.2.1. Let $G / H$ be a homogeneous space admitting a $G$-invariant $\tilde{G}_{2}$ structure. We assume that $G$ is a connected compact Lie group and $G$ acts effectively on $G / H$. Then $G / H$ is one of the following spaces

\begin{tabular}{|c|c|c|}
\hline Case & $G$ & $H$ \\
\hline 1 & $(S p(2) \times S p(1)) / \mathbb{Z}_{2}$ & $S O(4)_{3,4}$ \\
\hline 1 & $S O(5) \times S O(3)$ & $S O(4)_{3,4}$ \\
\hline $2 a i, \Gamma \subset \mathbb{Z}_{2} \times \mathbb{Z}_{2}$ & $S O(5)$ & $S O(3) \cdot \Gamma$ \\
\hline $2 c i, \Gamma \subset S p(1)$ & $S p(2)$ & $S p(1) \times \Gamma$ \\
\hline $2 c i, \Gamma \subset S O(3)$ & $S O(5)$ & $S p(1) \times \Gamma$ \\
\hline $2 c i i$ & $S U(3) \times T^{2}$ & $S U(2)$ \\
\hline $2 c i i$ & $P S U(3) \times T^{2}$ & $S U(2)$ \\
\hline 2aiii & $(S p(1) \times S p(1) \times S p(1)) / \mathbb{Z}_{2} \times U(1)$ & $S O(3)$ \\
\hline 2aiii & $S O(3) \times S O(4) \times U(1)$ & $S O(3)$ \\
\hline $2 a i i i$ & $S O(3) \times S O(3) \times S O(3) \times U(1)$ & $S O(3)$ \\
\hline $3 b i i,(k, l)=1, k \neq 0$ & $S O(5) \times U(1)$ & $S O(3) \cdot U(1)_{k, l}$ \\
\hline $3 b i i i,(k, l)=1, k l \neq 0$ & $S U(3) \times S U(2)$ & $S U(2)_{2,0} \cdot U(1)_{k, l}$ \\
\hline 3 biii $,(k, l)=1, k l \neq 0$ & $S U(3) \times S O(3)$ & $S U(2)_{2,0} \cdot U(1)_{k, l}$ \\
\hline $3 b i i i,(k, l)=1, k l \neq 0$ & $P S U(3) \times S U(2)$ & $S O(3)_{2,0} \cdot U(1)_{k, l}$ \\
\hline $3 b i i i,(k, l)=1, k l \neq 0$ & $P S U(3) \times S O(3)$ & $S O(3)_{2,0} \cdot U(1)_{k, l}$ \\
\hline 3aiii & $S U(3) \times S O(3)$ & $S O(3)_{2,3} \cdot U(1)_{1,0}$ \\
\hline 3aiii & $P S U(3) \times S O(3)$ & $S O(3)_{2,3} \cdot U(1)_{1,0}$ \\
\hline $4 i$ & $(S U(2) \times S U(2) \times S U(2)) / \mathbb{Z}_{2}$ & $T^{2}$ or $T^{2} \cdot \mathbb{Z}_{2}$ \\
\hline $4 i$ & $S O(3) \times S O(4)$ & $T^{2}$ or $T^{2} \cdot \mathbb{Z}_{2}$ \\
\hline $4 i$ & $S O(3) \times S O(3) \times S O(3)$ & $T^{2}$ or $T^{2} \cdot \mathbb{Z}_{2}$ \\
\hline $4 i i$ & $S U(3) \times U(1)$ & $U(1)_{k, k, k+1} \cdot U(1)_{m, m+1, m+1}$ \\
\hline $4 i i$ & $U(3)$ & $U(1)_{k, k, k+1} \cdot U(1)_{m, m+1, m+1}$ \\
\hline $4 i i$ & $P S U(3) \times U(1)$ & $U(1)_{k, k, k+1} \cdot U(1)_{m, m+1, m+1}$ \\
\hline $5 i$ & $S U(2) \times S U(2) \times U(1) \times U(1)$ & $U(1)_{k, k+1, l, l+1}$ \\
\hline $5 i$ & $S U(2) \times U(2) \times U(1)$ & $U(1)_{k, k+1, l, l+1}$ \\
\hline $5 i$ & $U(2) \times U(2)$ & $U(1)_{k, k+1, l, l+1}$ \\
\hline $5 i$ & $S O(4) \times U(1) \times U(1)$ & $U(1)_{k, k+1, l, l+1}$ \\
\hline $5 i$ & $S O(3) \times S O(3) \times U(1) \times U(1)$ & $U(1)_{k, k+1, l, l+1}$ \\
\hline $5 i i,(k, l)=1, k \neq l$ & $S U(3)$ & $U(1)_{k, l} \cdot \Gamma, \Gamma \subset U(1)$ \\
\hline $5 i i,(k, l)=1, k \neq l$ & $P S U(3)$ & $U(1)_{k, l} \cdot \Gamma, \Gamma \subset U(1)$ \\
\hline $5 i i, k=1=l$ & $P S U(3)$ & $U(1)_{1,1} \cdot \Gamma, \Gamma \subset S U(2)$ \\
\hline $6 i$ & $T^{7}$ & $\{e\}$ \\
\hline $6 i i$ & $S U(2) \times T^{4}$ & $\rho(H)=\{e\}$ \\
\hline $6 i i$ & $S O(3) \times T^{4}$ & $\rho(H)=\{e\}$ \\
\hline $6 i i i, \#(H)<\infty$ & $S U(2) \times S U(2) \times S^{1}$ & $\rho(H) \subset S O(3)_{3,3}$, or $\rho(H)=\mathbb{Z}_{k}$ \\
\hline $6 i i i, \#(H)<\infty$ & $S O(3) \times S U(2) \times S^{1}$ & $\rho(H) \subset S O(3)_{3,3}$, or $\rho(H)=\mathbb{Z}_{k}$ \\
\hline $6 i i i, \#(H)<\infty$ & $S O(3) \times S O(3) \times S^{1}$ & $\rho(H) \subset S O(3)_{3,3}$, or $\rho(H)=\mathbb{Z}_{k}$ \\
\hline
\end{tabular}

In this table, spaces have the same covering, if and only if they have the same numeration. We also use the notation $P S U(3)$ for the quotient $S U(3) / \mathcal{Z}(S U(3))$. 
We now define the degree of rigidity of $G / H$ as the dimension of the space of all $G$-invariant 3 -forms of $\tilde{G}_{2}$-type on $G / H$, and we denote this degree by $d_{3}(G / H)$. This dimension is equal to the dimension of the space of all $G$-invariant 3 -forms on $G / H$, since the $G L\left(\mathbb{R}^{7}\right)$-orbit of $\tilde{\phi}$ is open in $\Lambda^{3}\left(\mathbb{R}^{7}\right)^{*}$. Hence the degree of rigidity of $G / H$ equals the dimension of the space of all $\rho(H)$-invariant 3-forms on $V$.

We have the following decomposition (see e.g. [3], [28, table 5])

$$
\Lambda^{3}\left(V^{*}\right)=\Lambda_{1}^{3}\left(V^{*}\right) \oplus \Lambda_{7}^{3}\left(V^{*}\right) \oplus \Lambda_{27}^{3}\left(V^{*}\right),
$$

where $\Lambda_{i}^{3}\left(V^{*}\right)$ is the component of dimension $i$. The component $\Lambda_{1}^{3}$ is generated by $\tilde{\phi}$, the component $\Lambda_{7}^{3}$ is $\tilde{G}_{2}$-isomorphic (and hence $\rho(H)$-isomorphic) to $V^{*}=V$, and $\Lambda_{27}^{3}$ is $\tilde{G}_{2}$-isomorphic (and hence $\rho(H)$-isomorphic) to the space $S_{0}^{2}\left(V^{*}\right)$ of traceless quadratic forms on $V$. This isomorphism can be written explicitly as [4, $(2.15)]$

$$
i_{\tilde{\phi}}(\alpha \circ \beta)=\alpha \wedge *_{\tilde{\phi}}\left(\beta \wedge *_{\tilde{\phi}} \tilde{\phi}\right)+\beta \wedge *_{\tilde{\phi}}\left(\alpha \wedge *_{\tilde{\phi}} \phi\right) .
$$

Now let $\rho(H)$ be a subgroup of $S O(4)_{3,4} \subset \tilde{G}_{2} \subset G l\left(\mathbb{R}^{7}\right)$. Denote by $d_{1}$ the dimension of the fixed-point subspace of $V$ under the action of $\rho(H)$. Denote by $d_{2}$ the dimension of the subspace of all $\rho(H)$-invariant quadratic forms on $V$. Then we have

$$
d_{3}(G / H)=d_{1}+d_{2} .
$$

Dimension $d_{1}$ is already explicit from the embedding $\rho: H \rightarrow S O(4)_{4,3} \subset G l\left(\mathbb{R}^{7}\right)$. To compute $d_{2}$ we use the decomposition $S^{2}(\rho)$ computed in [28, table 5].

Remark 3.2.2. a) Since $S O(4)_{3,4}$ is also a compact Lie subgroup of $G_{2}$, all of the homogeneous spaces $G / H$ listed above also admit $G$-invariant $G_{2}$-structures. Hence the dimension of the space of all $G$-invariant 3 -forms on $G / H$ is at least 2 .

b) Some different spaces $G / H$ listed above are diffeomorphic as differentiable manifolds, e.g. $\left(S p(2) \times S p(1) / \mathbb{Z}_{2}\right) / S O(4)_{3,4}$ (case 1) and $S p(2) / S p(1)$ (case 2ci) are diffeomorphic to the standard sphere $S^{7}$. Other examples are the Wallach spaces in (5ii) with different $(\mathrm{k}, \mathrm{l})$. We refer the reader to [18, p. 466] for a precise formulation, when these Wallach spaces are diffeomorphic.

c) As a consequence of our classification we get a new proof for a statement in 20] that $S^{3} \times S^{4}$ admits no homogeneous $\tilde{G}_{2}$-structure. Since $S^{3} \times S^{4}$ is simply connected, by [23, Theorem A] if $S^{3} \times S^{4}$ admits a transitive action of a group $G$ it admits also a transitive action of a compact Lie subgroup $G^{\prime} \subset G$. On the other hand $S^{3} \times S^{4}$ is not in our list.

d) Clearly the dimension of the space of $G$-invariant $\tilde{G}_{2}$-structures on $G / H$ is equal to $d_{3}(G / H)-1$.

\section{Compact homogeneOUs MANiFolds ADMITting INVARIANT $G_{2}$-STRUCTURES}

In this section we classify all homogeneous spaces $G / H$ admitting a $G$-invariant $G_{2}$-structure such that $G$ is a compact Lie group and $H$ is a closed Lie subgroup (not necessary connected) of $G$. Our strategy is similar to that one in the previous section. We also compute the dimension of the space of $G$-invariant $G_{2}$-structures on $G / H$, see Remark 4.2.2 a. 
4.1. Reduction to a representation problem. We use the same method as in the previous section to classify all pairs $(H \subset G)$ of a compact Lie group $H$ in a compact Lie group $G$ such that $G$ acts effectively on $G / H$ and $G / H$ admits a $G$-invariant $G_{2}$-structure. First we will classify all pair of the corresponding Lie algebras $(\mathfrak{h} \subset \mathfrak{g})$ such that $\bar{\rho}(\mathfrak{h}) \subset \mathfrak{g}_{2}$. Combining the list of maximal Lie subalgebras in $\mathfrak{g}_{2}$ and the list of Lie compact subalgebras in $\mathfrak{s o}(4)_{3,4}$ in the previous section we get the following list of compact Lie algebras $\bar{\rho}(\mathfrak{h})$ in $\mathfrak{g}_{2}$

1) $\bar{\rho}(\mathfrak{h})=\mathfrak{s o}(4)_{3,4}$;

2) $\bar{\rho}(\mathfrak{h})=\mathfrak{s o}(3)$ with four possible embeddings into $\mathfrak{g}_{2}$. In the first three cases $(2 \mathrm{a})$, (2b), (2c) we have $\bar{\rho}(\mathfrak{h}) \subset \mathfrak{s o}(4)_{3,4}$, see also subsection 3.1. In the last case (2d) we have $\bar{\rho}(\mathfrak{h})=\mathfrak{s o}(3)_{7}$

3) $\bar{\rho}(\mathfrak{h})=\mathfrak{s o}(3)+\mathbb{R} \subset \mathfrak{s o}(4)_{3,4}$,

4) $\bar{\rho}(\mathfrak{h})=\mathbb{R}^{2}$

5) $\bar{\rho}(\mathfrak{h})=\mathbb{R}^{1}=\mathfrak{s o}(2)$ (there are infinitely many inequivalent embeddings of $\mathfrak{s o}(2)$ into $\mathfrak{s o}(4))$

6) $\bar{\rho}(\mathfrak{h})=0$

7) $\bar{\rho}(\mathfrak{h})=\mathfrak{g}_{2}$;

8) $\bar{\rho}(\mathfrak{h})=\mathfrak{s} u(3)$.

The first cases 1-5, except cases (2d), have been analyzed on the algebra level in the previous subsection 3.1. When lifting to the corresponding Lie subgroup we need to check whether the corresponding disconnected Lie subgroup $H$ belongs to $G_{2}$ but does not belong to $S O(4)_{3,4}$. Further, we notice that any subalgebra $\mathfrak{s} u(2)$ in $\mathfrak{s} u(3) \subset \mathfrak{g}_{2}$ is conjugate to $\mathfrak{s} u(2)_{0,4} \subset \mathfrak{s o}(4)_{3,4}$ or to $\mathfrak{s o}(3)_{3,3}$.

Proposition 4.1.1. Suppose that $\hat{G} / H$ admits a $\hat{G}$-invariant $G_{2}$-structure such that $\bar{\rho}(\mathfrak{h})$ is one of possibilities 1-5 listed above, except case (2d). Suppose that $\hat{G}=$ $G^{s c} \times T^{k}$ where $G^{s c}$ is a simply connected semisimple Lie group and $\bar{\rho}$ is a faithful representation. Then $(H \subset \hat{G})$ must be one of the pairs listed in Propositions 3.1.2, 3.1.4, 3.1.6, 3.1.7, 3.1.8, 3.1.9, Here we make the same assumptions for the cases considered in Propositions 3.1.7, 3.1.8 as in the previous section.

Proof. Let $H^{0}$ be the identity connected component of $H$. Since $H^{0} \subset S O(4)_{3,4}$ the space $\Lambda^{3}\left(\mathbb{R}^{3}\right)$ is invariant under the action of $H^{0}$ on $\Lambda^{3}\left(\mathbb{R}^{7}\right)$. Thus the invariance principle implies that $\rho(H) \subset G_{2}$, if and only if it belongs to $S O(4)_{3,4}=G_{2} \cap$ $(S O(3) \times S O(4))$. This completes the proof of Proposition 4.1.1

Let us consider the remaining cases. To handle the possibility (2d), we use our analysis in subsection 2.2. Case (2d) corresponds to case (2ii) with the associated embedding of $\mathfrak{s o}(3) \rightarrow \mathfrak{s o}(5)$ being a real irreducible representation of $\mathfrak{s o}(3)$ of dimension 5. Its connected subgroup in $\operatorname{Spin}(5)=S p(2)$ is the subgroup $S U(2)_{4}$, defined by the irreducible complex representation of $S U(2)$ of dimension 4 .

In possibility 6 , the argument in the previous section yields that there is no new case.

In possibility 7 , taking into account that $\operatorname{dim} \mathfrak{g}=21$, we conclude that $\mathfrak{g}=\mathfrak{s o}(7)$.

In possibility 8, taking into account that $\operatorname{dim} \mathfrak{g}=15$, we conclude that $\mathfrak{g}=\mathfrak{s} u(4)$ or $\mathfrak{g}=\mathfrak{g}_{2}+\mathbb{R}$.

Proposition 4.1.2. Let $\hat{G}=G^{s c} \times T^{k}$, where $G^{s c}$ is a connected simply-connected semisimple Lie group. Suppose that $\hat{G} / H$ admits a $\hat{G}$-invariant $G_{2}$-structure such that the action of $\hat{G}$ is almost effective. Suppose that $(H \subset \hat{G})$ is not listed in 
Proposition 4.1.1. Then $(H \subset \hat{G})$ is one of the pairs listed below:

Possibility (2d), $H=S U(2)_{4} \subset S p(2)$. The kernel of the action is $\mathcal{Z}(\hat{G}) \cap H=\mathbb{Z}_{2}$. Possibility (7), $H=G_{2} \cdot \Gamma \subset \operatorname{Spin}(7), \Gamma \subset \mathcal{Z}(\operatorname{Spin}(7))=\mathbb{Z}_{2}$. The kernel of the action is $\Gamma$.

Possibility (8), SU(3) $\cdot \Gamma \subset S U(4), \Gamma \subset \mathcal{Z}(S U(4))=\mathbb{Z}_{4}$. The kernel of the action is $\Gamma$.

Possibility (8), $S U(3) \cdot \Gamma \subset G_{2} \times S^{1}, \Gamma \subset \mathcal{Z}\left(G_{2} \times S^{1}\right)$. The kernel of the action is $\Gamma$.

Proof. It suffices to consider the case of disconnected Lie subgroups $H$. We have examined cases 1-6, except (2d). Applying Schur's Lemma to possibilities (2d) and (7), we conclude that $\mathcal{N}_{S p(2)}\left(S U(2)_{4}\right)$ is $S U(2)_{4}$ and $\mathcal{N}_{S p i n(7)}\left(G_{2}\right)=G_{2} \cdot \mathbb{Z}_{2}$. Applying the invariance principle to possibility 8 , we conclude that $\mathcal{N}_{S U(4)}(S U(3))=$ $S(U(3) \times U(1))$ and $\mathcal{N}_{G_{2} \times S^{1}}(S U(3))=\mathbb{Z}_{2}\left[D_{7}\right] \times S^{1}$. Using the decomposition $S(U(3) \times U(1))=S U(3) \cdot \mathcal{Z}_{S U(4)}(S U(3))$, we conclude that the isotropy action of an element $g \in \mathcal{N}_{S U(4)}(S U(3))=S(U(3) \times U(1))$ belongs to $G_{2}$, iff $g=g_{1} \cdot h$ where $g_{1} \in S U(3)$ and $h \in \mathcal{Z}(S U(4))=\mathbb{Z}_{4}$. Finally we check easily that $A d_{D^{7}}$ does not belongs to $G_{2}$, since $\left(S^{6} / \mathbb{Z}_{2}\right) \times S^{1}$ is not orientable. This proves Proposition 4.1 .2 .

4.2. Classification theorem. We summarize our examination in the previous subsection in the following

Theorem 4.2.1. Let $G / H$ be a homogeneous space admitting a $G$-invariant $G_{2}$ structure. We assume that $G$ is a connected compact Lie group and $G$ acts effectively on $G / H$. Then $G / H$ is one in Theorem 3.2.1 or one in the following list

$\begin{array}{ccc}\text { Case } & G & H \\ (2 d) & S O(5) & S O(3)_{5} \\ 7 & \operatorname{Spin}(7) & G_{2} \\ 7 & S O(7) & G_{2} \\ 8 & S U(4) & S U(3) \\ 8 & S U(4) / \mathbb{Z}_{2} & S U(3) \\ 8 & P S U(4) & S U(3) \\ 8 & G_{2} \times S^{1} & S U(3)\end{array}$

Remark 4.2.2. a) We have the same formula $d_{3}=d_{1}+d_{2}$ as in the case of $\tilde{G}_{2}$. The dimension of the space of all $G$-invariant $G_{2}$-structures on $G / H$ is $d_{3}(G / H)-1$. b) Many spaces among those listed in Theorem 4.2.1 have been known before. Case (2d) has been treated by Bryant in [3] and Bryant and Salamon in [5]. Case (5ii) has been examined by Cabrera, Monar and Swann [7. In 11] Friedrich and his coauthors classified all simply-connected compact homogeneous nearly parallel $G_{2^{-}}$ manifolds. We remark that a large part of homogeneous spaces listed in Theorem 4.2.1 are quotients of spaces listed in 11.

\section{Spaces $G / H$ With High RIGIDIty or WITH LOW RIGIDITY}

In this section we consider several examples of spaces $G / H$ with high rigidity or low rigidity. Many of these examples are known, but we provide simpler proofs of some known results based on our classifications. We also present some new results. 
5.1. Spaces $G / H$ with $d_{3}(G / H)=1$. Let $G / H$ be one of homogeneous spaces listed in Theorem 3.2.1 or Theorem 4.2.1. Clearly $d_{3}(G / H)=d_{1}+d_{2}$ is equal 1 , if and only if $d_{1}=0$ and $d_{2}=1$, so $G / H$ is in possibility (2d) or possibility (7). In other words invariant positive forms $\phi$ on these spaces are defined uniquely up to rescaling. These spaces are well studied before [3, 11]. They are nearly parallel $G_{2}$-manifolds, i.e.

$$
d \phi=\lambda * \phi
$$

for some constant $\lambda \neq 0$. We will give a brief explanation of this fact, which is close to the argument in [3. It is easy to see that equation (5.1) holds, because $d_{3}(G / H)=1$. To prove $\lambda \neq 0$, we observe that $d * \phi=0$, since there is no $\rho(H)$ invariant 2-form on $V$. On the other hand, by [1] there is no invariant metric with zero Ricci curvature on $G / H[1$. Hence $\lambda \neq 0$.

5.2. Spaces $G / H$ with $d_{3}(G / H)=2$. They are the spaces in possibilities (1), (2ci) with a nontrivial $\Gamma$, and in possibility (3aiii) listed in subsection 3.1. These spaces present an interesting class, since there are a 1-parameter family of inequivalent $G$-invariant $\tilde{G}_{2}$-structures on $G / H$, and a one-parameter family of inequivalent $G$-invariant $G_{2}$-structures on $G / H$.

An example of $\Gamma$ in the possibility (2ci) is the icosahedral rotation group of order 60 which is isomorphic to the alternating group $A_{5}$. The space is a quotient of a sphere $S^{7}$ by $\Gamma$.

Lemma 5.2.1. The dimension of the space of invariant 2-forms on $G / H$ with $d_{3}=2$ is less than or equal to 1. Any G-invariant 2-form on $G / H$ is closed.

Proof. The condition $d_{3}=2$ implies that $d_{1}=0$, since $d_{2} \geq d_{1}+1$. Now we use the following decomposition of $G_{2}$-modules, see e.g. 4]

$$
\Lambda^{2}\left(\mathbb{R}^{7}\right)^{*}=\mathfrak{g}_{2}+\mathbb{R}^{7} .
$$

Since $\rho(H) \subset G_{2}$, the above decomposition is invariant under the $\rho(H)$-action. Since $d_{1}=0$, the existence of a $G$-invariant 2 -form on $G / H$ is equivalent to the existence of a non-trivial centralizer $c$ of $\rho(H)$ in $\mathfrak{g}_{2}$. Thus either $\mathfrak{h}=\mathfrak{s} o(3)$, or $\mathfrak{h}=\mathfrak{s o} o(3)+\mathbb{R}$. In the first case, using our classification, we conclude that it is case (2cii) with $\mathfrak{h}=\mathfrak{s} u(2)_{0,4}$. Considering the decomposition of $\Lambda^{2}\left(\mathbb{R}^{7}\right)^{*}=\Lambda^{2}\left(\mathbb{R}^{3} \oplus \mathbb{R}^{4}\right)^{*}$ with respect to the representation of $\mathfrak{h}=\mathfrak{s} u(2)_{0,4}$, we conclude that there exists a vector in $\Lambda^{2}\left(\mathbb{R}^{3}\right)^{*}=\mathbb{R}^{3} \subset \mathbb{R}^{7}$, which is invariant under the action of $\rho(H)$. This contradicts our remark above that $d_{1}=0$. In the second case, since $r k \mathfrak{h}=2$, we conclude that $c$ lies in the component $\mathbb{R} \subset \mathfrak{h}$. In fact it is case (3aiii). This proves the first assertion of Lemma 5.2.1, and it gives rise to a unique (up to rescaling) $G$-invariant 2-form $\omega$ on $G / H$ as follows. We write $H=H_{0} \cdot U(1)$. Let us consider the $U(1)$-fibration $G / H_{0} \rightarrow G /\left(H_{0} \cdot U(1)\right)$ whose fiber is $\left.U(1) /\left(U(1) \cap H^{0}\right)\right)$. The form $\omega$ is the curvature of this non-trivial $U(1)$-fibration. Thus $\omega$ is a representative of a $G$-invariant 2 -form which is unique up to rescaling. Since it is closed, Lemma 5.2 .1 follows directly.

Theorem 5.2.2. Let $G / H$ be a compact homogeneous manifold with $d_{3}(G / H)=2$. a) Any $G$-invariant $\tilde{G}_{2}$-structure and any $G$-invariant $G_{2}$-structure on $G / H$ is coclosed.

b) There exists a unique $G$-invariant nearly parallel $G_{2}$-structure on $G / H$. 
Proof. a) It suffices to prove that $d \psi^{4}=0$, for any $G$-invariant stable 4 -form $\psi$. We will show that the pairing of $*_{\psi} d \psi^{4}$ with any $G$-invariant 2 -form $\omega$ is zero. This pairing is equal to the pairing of $\psi$ and $d \omega$. By Lemma 5.2.1 this pairing is zero.

b) The existence and uniqueness of a $G$-invariant nearly parallel $G_{2}$-structure on these spaces follows from a computation of the rank of a 4 -form $d \phi$, here $\phi$ is a $G$-invariant 3 -form on $G / H$, combining with the following observation. For all $G$-invariant 3 -forms $\phi$, all 4 -forms $d \phi$ are in the same conformal class. To prove this, we use the assertion that the dimension $d^{1}$ of the space of all 4 -forms $d \phi$ is equal to 1 , where $\phi$ is a G-invariant 3 -form on $G / H$. To see it, we note that $d^{1}$ is less than or equal to 2 . On the other hand, since the restriction of the Cartan form $\Omega^{3}$ to $V$ :

$$
\Omega^{3}(X, Y, Z)=\langle X,[Y, Z]\rangle
$$

is not zero on our spaces $G / H$ with $d_{3}=2$, and using $d \Omega^{3}=0$, we conclude that $d^{1} \leq 1$. A simple computation shows that $d^{1} \neq 0$. Hence $d^{1}=1$. Consequently, for all $G$-invariant 3 -forms $\phi$, all 4 -forms $d \phi$ are in the same conformal class.

Remark 5.2.3. The existence of $G$-invariant nearly parallel $G_{2}$-structures on spaces $G / H$ with $d_{3}=2$ and $\pi_{1}(G / H)=0$ has been established in [11] by a different method. In [16] Hitchin suggests a variational method to find nearly parallel $G_{2}$-structures.

Example 5.2.4. We consider case (1), see a detailed description in subsection 3.1. Using a method in [3] and [15] we explain how to find all $G$-invariant $\tilde{G}_{2}$-forms and $G$-invariant $G_{2}$-forms on $G / H$. Recall that $V=W+W^{\perp}$. Take an orthogonal basis $\left(e_{1}, e_{2}, e_{3}\right)$ in $W^{\perp}$. We choose another quaternion basis $e_{4}, e_{5}, e_{6}, e_{7} \in W$ with respect to the action of $\mathfrak{h}$. Let $e^{i}$ be the dual basis in $V^{*}$. Then the 3 -form $\omega^{123}$ and the 3 -form $\phi$ defined in Definition 2.2.1 are generators of our space of $\rho(H)$ invariant 3 -forms on $V$. The space of $\rho(H)$-invariant 4 -forms on $V$ is generated by

$$
\psi_{1}=\omega^{4567}, \psi_{2}=\omega^{4567}+\omega^{2367}+\omega^{2345}+\omega^{1357}-\omega^{1346}-\omega^{2356}-\omega^{1247} .
$$

Any $\rho(H)$-invariant 4 -form $\psi(a, b)$ on $V$ is of the form $a \psi_{1}+b \psi_{2}$. We define the associated 2-bilinear form $g_{\psi(a, b)}$ on $V^{*} \otimes V^{*}$ with value in $\left[\Lambda^{7}\left(V^{*}\right)\right]^{2}$ by setting [16, 8.4]

$$
g_{\psi(a, b)}\left(X^{*}, Y^{*}\right)=\left(X^{*} \wedge \psi(a, b)\right) \wedge\left(Y^{*} \wedge \psi(a, b)\right) \wedge \psi(a, b) .
$$

Since this is an invariant metric, and $d_{2}=2$, we calculate easily

$g_{\psi(a, b)}=\left(a^{2}(2 a+3 b)\left[\left(e_{1}\right)^{2}+\left(e_{2}\right)^{2}+\left(e_{3}\right)^{2}\right]+3 a^{3}\left[\left(e_{4}\right)^{2}+\left(e_{5}\right)^{2}+\left(e_{6}\right)^{2}+\left(e_{7}\right)\right)^{2}\right]\left(\omega^{1234567}\right)^{2}$.

Hence $\operatorname{vol}(\psi(a, b))=(a)^{3 / 2}(2 a+3 b)^{1 / 4}(3)^{1 / 3} \omega^{1234567}$. Thus $\psi(a, b)$ is a stable 4form, if and only if $a(2 a+3 b) \neq 0$. If $(2 a+3 b) a>0$ then $*_{\phi(a, b)} \phi(a, b)$ is a $G_{2}$-form, if $(2 a+3 b) a<0$ then $*_{\phi(a, b)} \phi(a, b)$ is a $\tilde{G}_{2}$-form.

5.3. Spaces with $d_{3}(G / H)=35$. It is easy to see that $d_{3}(G / H) \leq 35$, and the equality is attained, if and only if $H$ is trivial. On $G=T^{7}$ any $G$-invariant $G_{2^{-}}$ structure (or $\tilde{G}_{2}$-structure) is torsion-free. Now let us look at the next non-trivial case with $G=S U(2) \times T^{4}$ or $G=S O(3) \times T^{4}$.

Proposition 5.3.1. Let $G$ be $S U(2) \times T^{4}$ or $S O(3) \times T^{4}$.

(i) There is no $G$-invariant nearly $G_{2}$-structure on $G$.

(ii) There is no $G$-invariant closed stable 3-form on $G$. 
(ii) The dimension of the space of coclosed $\tilde{G}_{2}$-forms as well as the dimension of the space of coclosed $G_{2}$-forms on $G$ is 19 .

Proof. (i) The existence of a nearly $G_{2}$-structure implies that the associated metric is Einstein. By a theorem due to Alexeevskii and Kimeldeld [1, the Ricci curvature of the associated metric is positive, which implies that the fundamental group of $G$ is finite. So we obtain a contradiction.

(ii) Let us choose $e_{1}, e_{2}, e_{3} \in \mathfrak{s} u(2)$ such that $\left[e_{1}, e_{2}\right]=e_{2},\left[e_{1}, e_{3}\right]=-e_{3}$ and $\left[e_{2}, e_{3}\right]=e_{1}$. Let $e_{4}, e_{5}, e_{6}, e_{7} \in l T^{4}$, the Lie algebra of $T^{4}$. Suppose that there exists a closed stable 3 -form $\phi$. Assume that $\phi=c \omega_{123}+\phi_{0}$, where $\phi_{0}\left(e^{123}\right)=0$. Then $\left.\phi_{0}=d \psi^{2}+V_{1}\right\rfloor \omega^{4567}$, where $\psi^{2}$ is a $G$-invariant 2-form on $G$ and $V_{1} \in l T^{4}$. Since $d\left(e^{1} \wedge e^{2}\right)=0=d\left(e^{2} \wedge e^{3}\right)=d\left(e^{3} \wedge e^{1}\right)$, we can assume that $\psi^{2}$ has the form $a_{1} e^{1} \wedge f^{1}+a_{2} e^{2} \wedge f^{2}+a_{3} e^{3} \wedge f^{3}$ where $f^{i} \in\left(l T^{4}\right)^{*}$. Thus $\phi=c \omega^{123}+a_{1} e^{2} \wedge$ $\left.e^{3} \wedge f^{1}+a_{2} e^{1} \wedge e^{2} \wedge f^{2}+a_{3} e^{3} \wedge e^{1} \wedge f^{3}+V_{1}\right\rfloor \omega^{4567}$. Let $V_{2} \in l T^{4} \backslash\{0\}$ such that $f^{i}\left(V_{2}\right)=0$ for $i=\overline{1,3}$. If $V_{1}=b V_{2}$, then $r k(\phi) \leq 6$, so $\phi$ is not stable. If $V_{1}$ and $V_{2}$ are linearly independent, let us consider a space $\mathbb{R}^{6} \subset \mathfrak{g}$ containing $\left(e_{1}, e_{2}, e_{3}, V_{1}\right)$ which is a complement to $V_{2}$. Let $\theta$ be the 1 -form on $\mathfrak{g}$ such that $\theta\left(V_{2}\right)=1$ and $\theta_{\mid \mathbb{R}^{6}}=0$. Then $\phi=\theta \wedge \gamma_{1}+\gamma_{2}$, where $\gamma_{1} \in \Lambda^{2}\left(\mathbb{R}^{6}\right)^{*}$ has length 1 and $\gamma_{2} \in \Lambda^{3}\left(\mathbb{R}^{6}\right)^{*}$. By [2, Lemma 2], which reformulates results in [29], $\phi$ is not stable.

(iii) We set

$$
\phi_{ \pm}= \pm \omega^{123}+\omega^{145}+\omega^{167}+\omega^{246}-\omega^{257}-\omega^{347}-\omega^{356} .
$$

A simple calculation shows that $*_{\phi} \phi_{ \pm}= \pm \omega^{4567}+d \psi^{3}$, where $\psi^{3}=d\left(\omega^{167}+\omega^{145}-\right.$ $\left.\omega^{357}+\omega^{346}-\omega^{256}-\omega^{247}\right)$, hence $*_{\phi} \phi_{ \pm}$is a closed form. Clearly the dimension of the family of coclosed stable forms at $\phi_{ \pm}$is equal to $\operatorname{dim}\left(d \Omega_{G}^{3}\right)+5\left(=b_{4}(G)\right)$. Now we compute

$$
\begin{gathered}
\operatorname{dim}\left(d\left(\Omega_{G}^{3}\right)\right)=35-\operatorname{dim} \operatorname{ker} d_{\mid \Omega_{G}^{3},}, \\
\operatorname{dim}\left(\operatorname{ker} d_{\mid \Omega_{G}^{3}}\right)=5+\operatorname{dim}\left(d\left(\Omega_{G}^{2}\right)\right), \\
\operatorname{dim}\left(d\left(\Omega_{G}^{2}\right)\right)=21-\operatorname{dim} \operatorname{ker} d_{\mid \Omega_{G}^{2},}, \\
\operatorname{dim} \operatorname{ker}\left(d_{\mid \Omega_{G}^{2}}\right)=2+\operatorname{dim}\left(d\left(\Omega_{G}^{1}\right)\right)=5 .
\end{gathered}
$$

Thus the dimension of the space of stable 3 -forms at $\phi_{ \pm}$is 19 . Applying this argument to other stable invariant 3 -forms we complete the proof of Proposition 5.3 .1 .

Remark 5.3.2. All the spaces considered above admit stable closed 4 -forms. Using a method in [16 we can construct metrics with $\operatorname{Spin}(7)$-holonomy or metric with $\operatorname{Spin}(4,3)$-holonomy on the product of these spaces with an interval. Hitchin considered only $\operatorname{Spin}(7)$-holonomy, but his arguments are applied to the case of $\tilde{G}_{2}$-structure and $\operatorname{Spin}(3,4)$-holonomy.

Acknowledgement. We thank Sasha Elashivili, Ines Kath and Jiři Vanžura for helpful remarks. We thank the anonymous referees for helpful suggestions and corrections, which improve our note greatly. H.V.L. is supported in part by Grant of ASCR IAA100190701. A part of this paper has been done while H.V.L. was visiting the ASSMS, GCU Lahore-Pakistan, and the University of Toulouse. She thanks these institutions for their hospitality and financial support. M.M. is supported in part by HEC of Pakistan.

Note added in proof. We thank Ines Kath for showing us a paper by F. Reidegeld [26], which partially overlaps with our results. 


\section{REFERENCES}

[1] D. V. Alekseevskit And B. N. Kimelfeld: Structure of homogeneous Riemannian spaces with zero Ricci curvature, Funktional Anal. i Prilozen 9 (1975), N 2, 5-11.

[2] J. Bureš And J. VAnžUra, Multisymplectic formes of degree three in dimension seven, Suppl. Rend. Circ. Mat. Palemo, Serie II, Numero 71 (2003), 73-91.

[3] R. Bryant: Metric with exceptional holonomy, Ann. of Math. 126(1987), 525-576.

[4] R. BRyant: Some remarks on $G_{2}$-structures, Proc. of Goekova Geometry-Topology Conference 2005, Goekova Geometry-Topology , Goekova 2006.

[5] R. Bryant and S. Salamon: On the construction of some complete metrics with exceptional holonomy, Duke Math Journal, 58 (1989), N3, 829-850.

[6] S. Chiossi And A. Fino: Conformally parallel $G_{2}$-structure on a class of solvmanifolds, Math. Z. 252 (2006), 825-848.

[7] F.M. Cabrera, M.D. Monar, A. Swann: Classification of $G_{2}$-Structures, J. of London Math. Soc. (2) 53 (1996),n.2, 407-416.

[8] R. Cleyton and A. Swann: Cohomogeneity one $G_{2}$-structures, Journal of Geometry and Physics, 44,(2002), 202-220.

[9] S. Donaldson and E. Segal: Gauge theory in higher dimension, II, arXiv:0902.3239

[10] E. B. Dynkin: The maximal subgroups of classical groups, Tr. Mosk. Mat. Obshch. 1 (1952), 39-166, translated in Amer. Math. Soc. Transl. (2) 6, (1957), 245-378.

[11] Th. Friedrich, I. Kath, A. Moroianu, U. Semmelmann: On nearly parallel $G_{2^{-}}$ structures, J. of Geometry and Physics, 23 (1997), 259-286.

[12] J. Gauntlett, D. Martelli, S. Pakis: Superstrings with intrinsic torsion, Phys. Rev. D69, 086002, (2004).

[13] R. Harvey and H.B. Lawson: Calibrated geometries, Acta Math.,148, (1982), 47-157.

[14] S. Helgason: Differential Geometry, Lie groups Symmetric spaces, Academy Press, (1978).

[15] N. Hitchin: The geometry of three forms in six and seven dimensions, J. Diff. Geom. $55(2000), 547-576$.

[16] N. Hitchin: Stable form and special metrics, Contemp. Math. 288(2001), 70-89.

[17] P. Ivanov and S. Ivanov: $S U(3)$-Instantont and $G_{2}$, Spin(7)-Hetoric String Solitons, Comm. Math. Phys. 259, 79-102 (2005).

[18] M. KRECK AND S. Stolz: Some non-diffeomorphic homeomorphic homogeneous 7manifolds with positive sectional curvature, J.D.G. 33(1991), 465-486.

[19] I. KAтH: $G_{2(2)}^{*}$-structures on pseudo-Riemannian manifolds, J. Geometry and Physics 27 (1998), 155-177.

[20] H. V. LE: The existence of closed 3-forms of $\tilde{G}_{2}$-type on 7-manifolds, arXiv:math/0603182

[21] H. V. LE: Manifolds admitting a $\tilde{G}_{2}$-structure, arXiv:0704.0503 1 (the first version).

[22] H.V. Le, M. Panak, J. Vanzura: Manifolds admitting stable forms, CMUC 49 (2008), N1, 101-117.

[23] D. Montgomery: Simply connected homogeneous spaces, Proc. of AMS, vol.1, N4, (1950), 467-469.

[24] A. L. Onнishснiк: Lectures on Real Semisimple Lie algebras and Their Representations, EMS, 2004

[25] W. ReIchel: Über trilineare alternierende Formen in sechs und sieben Veränderlichen und die durch sie definierten geometrischen Gebilde, Dissertation Greiswald, (1907).

[26] F. Reidegeld, Spaces admitting homogeneous $G_{2}$-structures, D.G.A. 28(2010), 301-312.

[27] S. M. Salamon, Riemannian geometry and holonomy groups, Pitman Research Notes in Mathematics, vol. 201, Longman, Harlow, 1989.

[28] E. B. Vinberg And A.L. Onhishchik: Lie groups and algebraic groups, Moscow, Nauka (1988), English translation in Springer Series in Soviet Mathematics, Springer-Verlag, Berlin, 1990.

[29] R. Westwick: Real trivectors of rank seven, Linear and multilinear algebra, 10, (1981), 183-204

Address: Mathematical Institute of ASCR, Zitna 25, CZ-11567 Praha 1, email: hvle@math.cas.cz and ASSMS, Government College University, Lahore-Pakistan, email:mobeenmunir@gmail.com 